\title{
Large Games with a Bio-Social Typology*
}

\author{
M. Ali Khan ${ }^{\dagger}$, Kali P. Rath ${ }^{\ddagger}$, Yeneng $\mathrm{Sun}^{\S}$, and Haomiao Yu
}

\begin{abstract}
We present a comprehensive theory of large games in which players have names and determinate social-types and/or biological traits, and identify through four decisive examples, essentially based on a matching-pennies type game, pathologies arising from the use of a Lebesgue interval for player's names. In a sufficiently general context of traits and actions, we address this dissonance by showing a saturated probability space as being a necessary and sufficient name-space for the existence and upper hemi-continuity of pure-strategy Nash equilibria in large games with traits. We illustrate the idealized results by corresponding asymptotic results for an increasing sequence of finite games.

(100 words)
\end{abstract}

Keywords: Large games, social-type, traits, idealized limit game, saturated probability space, pure-strategy Nash equilibrium, closed-graph property, upper hemi-continuity, asymptotic implementation

JEL Classification Numbers: C62, D50, D82, G13.

\footnotetext{
* Some of the results reported here were presented at the Fall 2005 Meetings of the Midwest Economic Theory Group held in Lawrence, Kansas, October 14-16, 2005; at the Far East and South Asian Meeting of the Econometric Society in Singapore, July 16-18, 2008; at Professor In-Koo Cho's seminar on February 18, 2009 at the University of Illinois at Urbana-Champaign; and at the IMS Workshop on Probabilistic Impulse in Modern Economic Theory on January 11-18, 2011 at the National University of Singapore. The authors thank George Akerlof and Steven Durlauf for encouragement and correspondence. This version took its present form when Sun visited Ryerson University in August/September 2012. The authors also thank the Departments of Economics at Johns Hopkins and NUS for supporting visits in January 2010 (Sun), June 2010 (Khan) and June 2011 (Yu). This research has also been supported by the Social Sciences and Humanities Research Council of Canada (SIG program) and Ryerson University.

${ }^{\dagger}$ Department of Economics, The Johns Hopkins University, Baltimore, MD 21218, USA.

${ }^{\ddagger}$ Department of Economics, University of Notre Dame, Notre Dame, IN 46556, USA.

$\S$ Department of Economics, National University of Singapore, 1 Arts Link, Singapore 117570.

๑Department of Economics, Ryerson University, Toronto, ON M5B2K3, Canada.
} 


\section{Introduction}

Under the assumption that a player's payoffs depend, in addition to her own action, on a statistical summary, be it an average or a distribution, of the plays of everyone else in the game, the basic thrust of the theory of large games is its focus on pure-strategy equilibria. ${ }^{1}$ Indeed, this constitutes the raison d'etre of the theory and is easily justified by virtue of the fact that pure-strategy equilibria do not necessarily exist in games with a finite set of players. ${ }^{2}$ The two distinguished, and defining, features of the theory are a player's numerical negligibility and her societal interdependence in the original Nash formulation being substituted by more composite and aggregate measures of the actions of everyone else in the game. Within such a rubric, results on the existence of pure-strategy equilibria, as well as their asymptotic implementability and invariance to permutations of names, have been established. ${ }^{3}$ In addition, issues concerning measurability, purification and symmetrization have been identified and resolved in terms of decisive counterexamples and attendant theorems. The resulting theory has been shown to hinge on the cardinality of the underlying action set: if it is a finite, ${ }^{4}$ or a countably infinite, set, ${ }^{5}$ numerical negligibility of an individual player can be successfully formalized by an arbitrary atomless probability space; if, on the other hand, it is uncountable and compact, say even the unit interval, an arbitrary probability space does not suffice, and additional structure has to be put on the formalization of agent multiplicity. Initially, such a structure was invoked through the consideration of an atomless Loeb probability space as in [22], but recent work has identified a crucial property, namely saturation, and shown that agent multiplicity formalized by saturated probability spaces, ${ }^{6}$ a more general class to which atomless Loeb probability spaces belong, is not only sufficient but also necessary for the results to hold. We have thereby a viable and robust theory of large games.

A technical point of departure for the theory is the fact that the players' names do not have a natural "measure of closeness" defined on them, and that therefore the space of such names has to be conceived as an abstract rather than a topological measure space. It is by now well-appreciated that this space has necessarily to be endowed with an abstract measurable structure rather than a Borel or Baire structure that is metrically-, or more generally, topologically-generated. From a substantive point of view however, this has led to a theory that, in its most basic form, is informationally sparse. It is sparse in the sense that each agent, ${ }^{7}$ in taking the distribution of the societal actions as given, is focussed only on the proportions of agents playing certain actions, and oblivious to any social-types ${ }^{8}$ or social or biological traits by which the players could also be distinguished. This is to say that the theory, as currently formulated, ignores players' traits more generally conceived. Thus, in the context of traffic moving through a bridge or a tunnel,

\footnotetext{
${ }^{1}$ For details as to terminology and bibliographic substantiation of all claims in the ensuing paragraph, see the survey chapter [23] and its references.

${ }^{2}$ The matching-pennies game is a canonical, though by no means the only, example in this regard.

${ }^{3}$ See [22] where the invariance to permutations is labeled as the homogeneity property.

${ }^{4}$ Various results related to pure-strategy Nash equilibria in the case of finite actions follow from a general purification principle formulated by Dvoretzky-Wald-Wolfowitz in early 1950s; see [20].

${ }^{5}$ See [36] for the relaxation of the compactness assumption on action sets in [21].

${ }^{6}$ We have stated two characterizations for the reader's convenience as Propositions A and B below. See [11] and [17] for applications of saturated probability spaces in probability theory, theory of correspondences and game theory.

${ }^{7}$ In the sequel, and especially in the informal discussion, we shall use "agent" interchangeably with "player," and "society" interchangeably with "all the players."

${ }^{8}$ Note that we are avoiding the word type on its own, using it always with its hyphenated counterpart. This is being done to keep away from the notion of Harsanyi types that refers primarily to player beliefs. As elaborated below, we have the sociological and, older biological, pre-game-theory usage in mind; the reader can see, for example, the first essay in [14] and track his use of the word type, and note that we use the word trait in conformity with his pre-Harsanyi notion of type. Hence also the composite bio-social typology in the title.
} 
an individual decision-maker deciding on a possible route, again as conceived by the theory, notes only the proportion of the traffic moving through one of the two alternative routes, and disregards as irrelevant to her decision all other information concerning traffic-traits, including the proportion of truck-drivers and the unruliness of driving patterns. In another illustrative context, in deciding which of two possible candidates to vote for, an agent, as conceived by the theory, is concerned only with the proportion of the electorate that votes for either of the candidates, and thereby neglects all other information relating to a voter's biological or socioeconomic traits, proxied by variables that may be continuous and/or discrete. In short, the theory ignores relevant social categories for the equilibrium outcome.

To be sure, even the very early extensions of the theory did consider situations where the space of players' names could be conceived as being finitely partitioned, and an agent's preferences taken to depend on the mean or the distribution of the profile of societal actions when this profile is restricted to each individual element of this partition. ${ }^{9}$ Indeed, recent work has shown that the results can be extended to partitions with a countably infinite set of elements. This is of course important in that in removing the finiteness assumption, it removes an arbitrary and uniform bound on the grouping of agents. ${ }^{10}$ There are sublists of names, finite or countably infinite, according to which a continuum of agents is grouped, and an agent is dependent on society's plays only to the extent that she is dependent on the summary statistics pertaining to the individuals in each of these exogenously classified sublists or subgroups. However, nothing is said as regards how such sublists are determined, which is to say that the grouping of names is not tethered to observable and quantitatively measurable bio-social traits of the players. The players within the same subgroup can be interpreted as having the same social-type. However, there could be cases where the number of distinct social-types is not discrete. For example, the payoffs may depend on societal summary statistics that involves a continuum of socioeconomic traits, possibly income levels in the voting context, or vehicletonnage in the traffic example. For example, markets fail in situations of adverse selection precisely when a player is unwilling to subsidize agents perceived by her to be ranked "below" her traits. Whether this perception is "objective" or universally subscribed to by all of the players in the game is hardly relevant; it figures in each individual's decision. To pursue the point a little more, rather than a finite or a countable infinite list of exogenous classifications, one wants to deal with situations when the particular subset of society whose summary statistics are seen as relevant for an agent's decisions, is decided on by the agents' socioeconomic traits in the group, possibly income levels in the voting context, or vehicle-tonnage in the traffic example.

Thus, inspite of the useful earlier extensions, the theory remains, what we are characterizing here as being, informationally sparse. The question then is whether a model can be formulated to cover situations where an agent, in deciding on her individual action, has available to her comprehensive information not only on what players in a particular sublist are playing, but also on the variety of social-types or biological and other traits associated with the players in that sublist, as well as some conception of the social group she belongs to and the type of traits she shares. This is to ask, in other words, whether the theory can be generalized to take into account the fact that a player has a name as well as a social-type or trait, ${ }^{11}$ the

\footnotetext{
${ }^{9}$ This case of a finite partition goes back to Remark 2 in [32]; for subsequent work, see the references in [23] and subsequent to that survey, in [36].

${ }^{10}$ See Chapter 5 in [12], and note that his dissertation presents an extension in the context of both countable and uncountable compact metric action sets.

${ }^{11}$ As has already been emphasized above in Footnote 8, we are using social-type and trait as synonymous terms. As we shall see in the sequel, we use characteristic as a more general term that also covers a player's payoffs.
} 
former being chosen from an abstract probability space $(I, \mathcal{I}, \lambda)$, the latter from a complete separable metric space $T$, and with a deterministic (measurable) function $\alpha$ connecting each element of one to the other. This connection of a social-type or trait to an individual name by $\alpha$ is one of the essential constituents of the data of the large game in our reformulation. The essence of the reformulation then is to conceive of the summary of societal actions as distributions on the product space of actions and traits such that their marginal distribution on the space of traits is always identical to the given distribution of traits of the game, this distribution $\rho$ being induced on the trait space $T$ by the function $\alpha$. This allows an individual player access to information based on traits as well as on proportions, and with individual payoffs depending on the action set, on the one hand, and on an extended space of externalities on the other. One can now formally define a large game and its Nash equilibrium in pure strategies, and proceed with an investigation of these reformulated objects. It is precisely such a model that is being offered in this paper.

However, before getting into the technical difficulties of such an extension, it is important to note that our basic motivation, coming as it does from the theory of large games, dovetails into the rich theoretical and econometric work on identity and social interactions pioneered by Akerlof-Kranton [1]. ${ }^{12}$ They abstract their paper on "economics and identity" with the following words.

This paper considers how identity, a person's sense of self, affects economic outcomes. In the utility function we propose, identity is associated with different social categories and how people in these categories should behave. We then construct a simple game-theoretic model showing how identity can affect individual interactions.

The point is that the reformulation that we study lifts the Akerlof-Kranton conception from the setting of a finite game to that of a large game, and even though there are important differences between their conception and the one studied in this paper, there is an undeniable complementarity. Thus, there are passages in [1] where their context is clearly that of a "large" society; see, for example, their "identity model of poverty and social exclusion" in which they conceive of a "a large community, normalized to size one, of individuals (page 740)." As such, the model reported here can also be seen as presenting a rigorous formalization of their ideas. Consider, for example, the utility function of the $i^{\text {th }}$-player on which their analysis revolves:

$$
U_{i}=U_{i}\left(\mathbf{a}_{i}, \mathbf{a}_{-i}, I_{i}\right), \text { with } I_{i}=I_{i}\left(\mathbf{a}_{i}, \mathbf{a}_{-i}, \mathbf{c}_{i}, \epsilon_{i}, \mathbf{P}\right)
$$

where $\mathbf{a}_{i}$ is the $i^{t h}$-player's action, $\mathbf{a}_{-i}$ the actions of everyone else in the finite game, and the novel variable $I_{i}$ which "shows how identity can be brought into economic analysis, allowing a new view of many economic problems." This "new type of externality" $I_{i}$ depends on the particular social category $\mathbf{c}_{i}$ chosen from a set of exogenously-given categories $\mathbf{C}$, and on how the player's "own" given trait $\epsilon_{i}$ match the ideal of $i$ 's assigned category indicated by the exogenously-given prescriptions $\mathbf{P}$. In the context of the model of this paper, this "new type of externality" is given by the inclusion of $\tau$ in an individual player's utility function

$$
U_{i}=U_{i}(a, \tau) \text { with } \tau=\lambda(\alpha, f)^{-1} \in \mathcal{M}^{\rho}(T \times A),
$$

where $\tau$ is a probability measure on actions and traits analogous to the individual identity variable $I_{j}$, and one which is endogenously obtained as a distribution of the Nash equilibrium $f$ taking names into actions. In

\footnotetext{
${ }^{12}$ The reader can also see the subsequent surveys in [7] and [15], and their rich and diverse bibliographies.
} 
keeping with our large-game formulation, only a summary of societal actions play a role. There is of course more to be said in terms of a comparison between the two models, and we shall do so in the sequel.

The fact is that the extension of the theory to the model discussed in the above paragraph is not straightforward, and as shown in Example 1 below, there is no Nash equilibrium in a large game in which the set of players' names are formalized as an arbitrary, atomless probability space with the unit interval $I$ as the space of traits even when the cardinality of the common action set is two! In particular, Example 1 below considers a large game of matching pennies with balanced players, where the space of names is modeled as the Lebesgue unit interval and with the $i^{\text {th }}$ player who always tries to balance out those players younger than her in the sense that if there are more younger players who play Heads (Tails), then player $i$ will play Tail (Head). With this counterexample, it is clear that additional assumptions will have to be made on the model if the cardinality of the space of players' traits is not to be restricted.

It is worth emphasizing that the significance of this example for the theory of large games does not end with it closing the door to the existence of a pure-strategy Nash equilibrium in situations where players have names as well as traits, and the space of players' names is formalized as any abstract probability space; to wit, the Lebesgue unit interval. It raises an equally interesting issue when discretized and cast in the form of a sequence of games with a large but finite set of players. It is now well understood that in general a discretized sequence of finite games has only an approximate equilibria in pure strategies, with the approximation becoming finer as the number of players becomes larger. ${ }^{13}$ What is interesting in the sequence of finite-player games which is a discrete version of the game in Example 1 is that each of its elements has an exact Nash equilibrium in pure strategies; see Example 2 below! It is thus clear that additional assumptions will have to be made on the space of players' names, and the example is decisive in establishing that an arbitrary atomless probability space simply will not do.

The technical point of departure for this paper is that this additional assumption in the requirement that the probability space of players' names not only be atomless but that it be saturated in the specific sense that the attendant $\sigma$-algebra, when restricted to any non-negligible set, not be countably-generated. The property shifts the emphasis from the cardinality of the space of player-names to the cardinality of the space of playercoalitions ${ }^{14}$ by ensuring that all the subspaces are rich in measurable sets. It is thus simple enough that it can be verbally stated even in an introduction oriented to a general audience. As a natural notion in theory of large games, saturated probability spaces were first applied to game theory in [17, Theorem 4.6], where it was shown that an atomless player space is saturated if and only if every game based on an uncountable compact metric action space has a Nash equilibrium.In hindsight, it is precisely the fact that an atomless Loeb space is saturated that was responsible for the robust viability of the theory of large games with a compact metric action set structured on Loeb spaces, and its sufficiency claims were now complemented and completed by assertions pertaining to necessity. ${ }^{15}$ The fact that the Lebesgue unit interval does not satisfy this saturation property then emerges as rather routine anti-climax. In any case, with the assumption

\footnotetext{
${ }^{13}$ This is precisely the question of asymptotic implementability of an idealized limit game; see [23] for a discussion. If specific speeds of convergence are required, which is to say, the determination of the error for an arbitrarily given finite game, see [28]. These alternative ways of formalizing a particular kind of regularity for systems with a continuum of agents are now well-understood.

${ }^{14}$ It is worth emphasizing that being in the register of non-cooperative game theory, the word coalition is being used in a purely technical and non-substantive sense of collections or ensembles of players.

${ }^{15}$ See [22]; we refer here of course to the available theory, one that does not take into account the reformulation that is being pursued in this paper. The fact that the results generalize to include the reformulation is of course one of the principal objectives of this paper.
} 
of a saturated probability space, and with the counterexample thereby bypassed and made irrelevant, one can proceed with a basic extension of the theory. Towards this end, we show that saturated probability spaces are necessary and sufficient for the existence of pure-strategy equilibria in games with traits. These "necessity results," in emphasizing the irrelevance to the existence theory of pure-strategy Nash equilibria of all probability spaces which have countably-generated subspaces, leave alone Lebesgue spaces, are important and new to this paper. ${ }^{16}$

However, even if the door to the existence issue is closed by saturated probability spaces, the issue of continuity remains to be considered. Theorems 1 and 2 below pertain to the question of the existence of a pure-strategy Nash equilibria of games with traits, and therefore affirmatively answer the question of the equilibrium correspondence being well-defined in the sense of having non-empty values. The unanswered question is the continuity of this correspondence. Example 2 concerns a sequence of games motivated by Example 1 without an equilibrium but with each member of the sequence with an equilibrium. We supplement this example by Example 3 which keeps this feature but also ensures that the sequence of games converges to the idealized game of Example 1. Thus, an arbitrary atomless measure space of players' names, the Lebesgue unit interval in particular, is again revealed to be inappropriate. How is this dissonance to be resolved? Not unsurprisingly, the answer is a saturated probability space. In Example 4, we consider a variant of Example 1 in terms of player spaces in which the Lebesgue interval is substituted to its saturated extension, and to which the sequence of games in Example 3 converges. As far as this specific example is concerned, it represents a trial-run and suggests a complete characterization of saturated spaces in terms also of the closed-graph property. This is then realized by Theorems 3 and 4, paralleling Theorems 1 and 2 , in affirmatively answering the question of the equilibrium correspondence possessing the closed-graph property. The first of these gives a sufficient condition for the property, and the second characterization result not unlike Theorem 2. It is well understood in game theory, at least since Roberts-Postlewaite [31] that is the lower hemi-continuity of the equilibrium correspondence that is the source of difficulty, and that upper hemi-continuity or the closed-graph property is routine. ${ }^{17}$ This is why Example 3 is interesting: it questions the prevailing intuition; and the interest of Theorems 3 and 4 then lies in their appeal to the saturation property to re-establish it.

Thus far, for a large game with traits, we have Theorems 1 to 4 that cater both to the existence and continuity properties of pure-strategy Nash equilibria. For a comprehensive and well-rounded theory, this then leads to the question of the relevance of these results to a finite-agent setting, in the first instance, and to a large but finite set-up in the second. In short, it leads to the standard methodology for asymptotic implementation, with or without speeds of convergence, of the limit results. ${ }^{18}$ However, it is worth emphasizing that unlike this literature, our focus is not only on existence issues but also on those pertaining to continuity. It is this that leads us to obtain Proposition 1 as an approximate version of the conclusion of Theorem 3 by avoiding these procedures, and by simply modifying the proof of the latter. It is in the context

\footnotetext{
${ }^{16}$ As discussed in [24], one cannot simply write off Lebesgue spaces, or their extensions going back to an early paper of Kakutani [16]. However, even though these extensions have some role to play, the necessity results in [17], and in this paper, show that they cannot really close the breach opened by the counterexample. As long as the player space is non-saturated, there must exist a large game with traits that has no equilibrium in pure strategies.

${ }^{17}$ Thus in the discussion of their example, with $Q$ being their equilibrium correspondence, Roberts-Postlewaite [31, p.124] write "[If] we consider the limit $\mu$ of the sequence $\left\langle\mu^{k}\right\rangle, Q(\mu)$ contains all prices which which are normals of planes. Thus $Q$ "blows up" at the limit: it is upper hemi-continuous at $\mu$ but not lower hemi-continuous."

${ }^{18}$ This is one of the three criteria for a viable name space that [22] adduce in favor of Loeb spaces.
} 
of Proposition 2, a translation of Theorem 1(ii), that we turn to the by-now conventional ${ }^{19}$ methodology for asymptotic implementation of an exact result on Loeb spaces. The fact that the saturation property of a probability space is shared by atomless Loeb probability spaces, and therefore, by Loeb counting spaces, allows this methodological procedure to be activated in the service of what can also be seen as a weak form of a lower hemi-continuity property.

The rest of the paper is organized as follows. We confine Section 2 to a parsimonious description of the reformulation of a large game that has been informally described above, and show how it includes earlier formulations of large games based on an exogenously-given finite or countably infinite partitions of the space of player's name. From both the technical and conceptual points of views, it is the formulation of societal dependence, or externalities in the economic theory jargon, that sets the stage for the entire analysis to follow. In Section 3, we show the existence of a pure-strategy Nash equilibria under the hypothesis that the space of players' names is a saturated probability space and the space of traits is a complete separable metric space, taking care to emphasize that the assumption of a saturated probability space is necessary. In Section 4, we present four examples, and it is important for the reader to appreciate how they relate to each other: the basic matching-pennies-game intuition of Examples 1 and 2 is used as point of departure for Example 3 focused on the convergence of the sequence of games, and Example 4 used to resolve dissonances between Example 1 and the other two. In Section 5, we turn to the closed graph property of a Nash equilibrium correspondence, and in Section 6, to an asymptotic implementation of both the existence and closed graph properties in terms of approximate equilibrium concepts. Section 7 concludes the paper. In the Appendix, we collect for the reader's convenience some relevant results from the earlier literature, and also relegate to it some rather technical results ${ }^{20}$ that are required in Sections 3 and 4.

\section{A Reformulation}

It is by now conventional to see a large game as being constituted by two basic objects: an abstract atomless probability space $(I, \mathcal{I}, \lambda)$ representing the space of player names, and a compact metric space $A$ representing a common action space. The common action space is then used to build a space of payoff characteristics, thereby leading to a definition of a game and its (pure-strategy) Nash equilibrium. When endowed with its Borel $\sigma$-algebra, the action set leads to the Borel measurable space $(A, \mathcal{B}(A))$, and through it, to the space $\mathcal{M}(A)$ of all probability measures on $A$ endowed with its weak topology. ${ }^{21}$ The space $\mathcal{M}(A)$ is then also a compact metric space, and it represents the distributions of possible plays in the game. In terms of the vocabulary used in the introduction, it represents "externalities" or "society's plays". The space of players' payoffs $\mathcal{U}_{A}$ is then given by the space of all continuous functions on the product space $(A \times \mathcal{M}(A))$, and based on its sup-norm topology and endowed with its resulting Borel $\sigma$-algebra, it can also be conceived as a measurable space $\left(\mathcal{U}_{A}, \mathcal{B}\left(\mathcal{U}_{A}\right)\right)$ of players' characteristics. Note that the space of players' names $I$ does not figure in the space of players' characteristics. A large game is then a random variable in $\mathcal{U}_{A}$ and its Nash equilibrium a random variable in $A$. More formally,

Definition 1. A large game is a measurable function $\mathcal{G}^{0}$ from $I$ to $\mathcal{U}_{A}$. A Nash equilibrium of a game $\mathcal{G}^{0}$ is

\footnotetext{
${ }^{19}$ See $[22]$ and its references. Also see the qualifier in Footnote 36.

${ }^{20}$ We do not mean to devalue these results, and shall indicate their novelty, and possible relevance for future work, when we get to them in the text itself.

${ }^{21}$ We conform to standard usage and forgo referring to this as the weak*-topology, the formally correct designation.
} 
a measurable function $f: I \longrightarrow A$, such that for $\lambda$-almost all $i \in I$, and with $u_{i}$ abbreviated for $\mathcal{G}^{0}(i)$,

$$
u_{i}\left(f(i), \lambda f^{-1}\right) \geq u_{i}\left(a, \lambda f^{-1}\right) \text { for all } a \in A
$$

All this is now standard as surveyed in [23].

The reformulation of a large game that we study in this paper rests on four, rather than two, basic objects: in addition to space of players' names $I$ and the common action set $A$, we work with a complete, separable metrizable (Polish) space $T$ representing a space of possible player traits, and endowed with a probability measure $\rho$ on the Borel $\sigma$-algebra $\mathcal{B}(T)$ induced by the topology on $T$. The game and its Nash equilibria are then built up from these four basic objects. The first important element in the reformulation is that the externalities or society's plays are now conceived as a probability measure on the product space $(T \times A)$, with the latter endowed with its product Borel $\sigma$-algebra, ${ }^{22}$ and such that the marginal of the measure on $T$ is identical to the given measure $\rho$ on $T$. Formally, let $\mathcal{M}(T \times A)$ be the space of Borel probability distributions on $T \times A$, and $\mathcal{M}^{\rho}(T \times A)$ be the subspace of $\mathcal{M}(T \times A)$ such that for any $\tau \in \mathcal{M}^{\rho}(T \times A)$, its marginal probability on $T, \tau_{T}=\rho$. Note that unlike the space $\mathcal{M}(A)$, the space $\mathcal{M}(T \times A)$ is not necessarily compact in the weak topology. However it is a standard result that the space $\mathcal{M}^{\rho}(T \times A)$ is indeed compact.

Now, just as in the conventional theory described in the first paragraph of this section, the space of players' payoffs $\mathcal{V}_{(A, T, \rho)}$ is then given by the space of all continuous functions on the product space $\left(A \times \mathcal{M}^{\rho}(T \times A)\right)$, and based on its sup-norm topology and endowed with its resulting Borel $\sigma$-algebra, it can also be conceived as a measurable space $\left(\mathcal{V}_{(A, T, \rho)}, \mathcal{B}\left(\mathcal{V}_{(A, T, \rho)}\right)\right)$ of players' payoffs. The point to be made here is that in the generalized setting with socioeconomic traits, this does not exhaust the set of player characteristics which is now defined as the product $T \times \mathcal{V}_{(A, T, \rho)}$. Note that here again the space of players' names $I$ does not figure in the space of players' characteristics. We can now present a reformulation of a large game that again involves a random variable, but one taking values in a richer target space.

Definition 2. A large game with traits is a measurable function $\mathcal{G}$ from $I$ to $T \times \mathcal{V}_{(A, T, \rho)}$ such that $\lambda \mathcal{G}_{1}^{-1}=\rho$, where $\mathcal{G}_{i}$ is the projection of $\mathcal{G}$ on its $i^{\text {th }}$-coordinate, $i=1,2$. A Nash equilibrium of a game $\mathcal{G}$ is a measurable function $f: I \longrightarrow A$, such that for $\lambda$-almost all $i \in I$, and with $v_{i}$ abbreviated for $\mathcal{G}_{2}(i)$, and $\alpha: I \longrightarrow T$ abbreviated for $\mathcal{G}_{1}$,

$$
v_{i}\left(f(i), \lambda(\alpha, f)^{-1}\right) \geq v_{i}\left(a, \lambda(\alpha, f)^{-1}\right) \text { for all } a \in A .
$$

On decomposing the target space $\left(T \times \mathcal{V}_{(A, T, \rho)}\right)$, we see that in our reformulation, a large game with traits, is really a pair of random variables, one a measurable function $\alpha=\mathcal{G}_{1}$ from $I$ to $T$ associating each player $i \in I$ with her traits, or rather an array of traits $\alpha(i) \in T$, and the other, a measurable function $\mathcal{G}_{2}$ associating each player $i \in I$ with her payoff $\mathcal{G}_{2}(i) \in \mathcal{V}_{(A, T, \rho)}$.

In the context of games with exogenously-given, finite or countably infinite, partitions of the space of names, the earlier work of [21, 22], and its generalizations in [12], has already been mentioned in the introduction. We conclude this section by showing how the reformulation presented above subsumes these efforts as it moves forward. Specifically, we show how conventional formulations available in the literature are special cases of Definition 2. The issue revolves around moving back and forth from the conventional form of a large game where the externality parameters are based on the Cartesian product of probability

\footnotetext{
${ }^{22}$ Since we are assuming separability everywhere, the product Borel $\sigma$-algebra is the same as the Borel $\sigma$-algebra of the product of the spaces $T$ and $A$.
} 
measures on the action space, the index of the product running across the index of a countable, possible infinite, partition of the space of names, to one where it is based on a joint probability measure on the space of traits and actions. Since the space of names and the common action set are shared by both formulations, this reduces to moving back and forth between the payoff functions.

Towards this end, let $\left\{t_{k}\right\}_{k \in K}$ be a list of all the elements of $T$, where $K$ is an index set which is at most countable. $^{23}$ We have to relate a function $v \in \mathcal{V}_{(A, T, \rho)}$ to another function $u \in \mathcal{U}_{A}^{T}$ where ${ }^{24} \mathcal{U}_{A}^{T}$ is the space of real-valued continuous functions on $A \times \prod_{t \in T} \mathcal{M}(A)$. Define a function

$$
\Phi: \mathcal{M}^{\rho}(T \times A) \longrightarrow \prod_{t \in T} \mathcal{M}(A) \text { such that } \Phi(\tau)=\left\{\mu_{t}\right\}_{t \in T}
$$

where for any $t$ in $T$,

$$
\mu_{t}(B)=\tau(\{t\} \times B) / \rho(t) \text { for all } B \in \mathcal{B}(A)
$$

Now consider the function

$$
\Psi: \prod_{t \in T} \mathcal{M}(A) \longrightarrow \mathcal{M}^{\rho}(T \times A) \text { such that } \Psi\left(\left\{\mu_{t}\right\}_{t \in T}\right)=\tau
$$

where for all $C \in \mathcal{B}(T \times A)$,

$$
\tau(C)=\sum_{t \in T} \rho(t) \mu_{t}\left(C_{t}\right), C_{t}=\{a \in A:(t, a) \in C\}
$$

It is now easy to check that $\Psi$ is the inverse of $\Phi$, and that therefore the latter is a bijective mapping. And since $\Phi$ takes a compact set into a compact set, we can claim that it is also a homeomorphism if we show that $\Phi$ is continuous. We turn to this.

Suppose $\left\{\tau^{m}\right\}$ weakly converges to $\tau^{0}$. Denote $\Phi\left(\tau^{m}\right)$ by $\left\{\mu_{t}^{m}\right\}_{t \in T}$ and $\Phi\left(\tau^{0}\right)$ by $\left\{\mu_{t}^{0}\right\}_{t \in T}$. Now for any $k \in K$, pick any $\mu_{t_{k}}^{0}$-continuity set $B \in \mathcal{B}(A)$. Then it is clear that $\left(\left\{t_{k}\right\} \times B\right)$ is a $\tau^{0}$-continuity set in $\mathcal{B}(T \times A)$, and hence $\tau^{m}\left(\left\{t_{k}\right\} \times B\right)$ converges to $\tau^{0}\left(\left\{t_{k}\right\} \times B\right)$, and hence $\tau^{m}\left(\left\{t_{k}\right\} \times B\right) / \rho\left(t_{k}\right)$ converges $\tau^{0}\left(\left\{t_{k}\right\} \times B\right) / \rho\left(t_{k}\right)$, and hence $\mu_{t_{k}}^{m}(B)$ converges to $\mu_{t_{k}}^{0}(B)$. The assertion of our claim that $\Phi$ is a homeomorphism is complete.

And now we can define a function $\bar{\Phi}: \mathcal{U}_{A}^{T} \longrightarrow \mathcal{V}_{(A, T, \rho)}$ such that, for all $a \in A$,

$$
\begin{gathered}
v(a, \tau)=\bar{\Phi}(u)(a, \tau)=u(a, \Phi(\tau)), \\
u\left(a,\left\{\mu_{t}\right\}_{t \in T}\right)=\bar{\Phi}^{-1}(v)\left(a,\left\{\mu_{t}\right\}_{t \in T}\right)=v\left(a, \Psi\left(\left\{\mu_{t}\right\}_{t \in T}\right)\right),
\end{gathered}
$$

which allow one to go back and forth from the conventional formulation to the one studied here.

\footnotetext{
${ }^{23}$ We assume that $T$ is endowed with discrete topology. Without loss of generality, assume that $\rho\left(\left\{t_{k}\right\}\right)>0$ for all $k \in K$.

${ }^{24}$ Note that Definition 1 , as presented above, deals with the case of $T$ being a singleton; its extension to the case when $T$ has a finite or a countably infinite set of elements is straightforward; see the end of this section for the payoffs in this general setting.
} 


\section{Saturated Probability Spaces and Existence Theorems}

We now turn to the issue of the existence of Nash equilibria of the idealized limit game with traits as formulated in the previous section. We shall show that the relevant condition for the atomless space of players' names is that it be a saturated probability space, and that such a requirement is both sufficient and necessary. We begin with the basic definition.

Definition 3. (1) A probability space is said to be countably-generated if its $\sigma$-algebra can be generated by a countable number of subsets together with the null sets; otherwise, it is not countably-generated. A probability space $(I, \mathcal{I}, \lambda)$ is saturated if it is nowhere countably-generated, in the sense that, for any subset $S \in \mathcal{I}$ with $\lambda(S)>0$, the restricted probability space $\left(S, \mathcal{I}^{S}, \lambda^{S}\right)$ is not countably-generated, where $\mathcal{I}^{S}:=\left\{S \cap S^{\prime}: S^{\prime} \in \mathcal{I}\right\}$ and $\lambda^{S}$ is the probability measure re-scaled from the restriction of $\lambda$ to $\mathcal{I}^{S}$.

(2) An atomless probability space $(I, \mathcal{I}, \lambda)$ is said to have the saturation property for a Borel probability measure $\nu$ on the product of Polish spaces $X \times Y$ if for every measurable mapping $f: I \rightarrow X$ which induces the distribution as the marginal measure of $\nu$ over $X$, then there is a measurable mapping $g: I \rightarrow Y$ such that the induced distribution of the pair $(f, g)$ on $(I, \mathcal{I}, \lambda)$ is $\nu$.

The saturation property of a probability space plays an essential role in [17], and its verbal statement is justified on account of its importance: it ensures that any Borel measure on a product of two complete, separable and metrizable (Polish) spaces, and any random variable taking values in one of these spaces and whose law is the marginal of the given measure on that space, there exists another twin random variable taking values in the other space such that the law of the pair of random variables is the given measure. The reason for linking the two definitions presented above is now furnished by the following result.

Proposition A. A probability space $(I, \mathcal{I}, \lambda)$ is saturated if and only if it has the saturation property for each Borel probability measure $\nu$ on the product of any two Polish spaces. ${ }^{25}$

A saturated probability space admits as measurable functions a richer variety of non-cooperative behavior. For example, the Lebesgue unit interval, i.e., the interval $[0,1]$ associated with the $\sigma$-algebra of Lebesgue measurable sets and the Lebesgue measure, is countably-generated probability space, and thus not a saturated probability space. In comparison, any atomless Loeb probability space is saturated.

We can now present our first principal result. ${ }^{26}$

Theorem 1. Every large game with traits $\mathcal{G}: I \longrightarrow T \times \mathcal{V}_{(A, T, \rho)}$ has a Nash equilibrium if either of the following two (sufficient) conditions hold:

(i) $T$ and $A$ are both countable spaces,

(ii) $(I, \mathcal{I}, \lambda)$ is a saturated probability space.

Proof: Given any $\tau \in \mathcal{M}^{\rho}(T \times A)$, let the best response set $B(i, \tau)$ of player $i$ be

$$
B(i, \tau)=\operatorname{argmax}_{a \in A} v_{i}(a, \tau),
$$

\footnotetext{
${ }^{25}$ This proposition is available in [11, Theorem 3B.7, page 47]. A version of that equivalence result was first noted in [13]. We also note that throughout the paper, we reference results previously available in the literature as "propositions" alphabetically ordered. Numerical ordered propositions, however, are new to this work.

${ }^{26}$ Note that we work with the standing hypothesis that the space of players' names $(I, \mathcal{I}, \lambda)$ is an atomless probability space, that the common action set $A$ is a compact metric space, and that $T$ is a Polish space endowed with a probability measure $\rho$.
} 
where $v_{i}=\mathcal{G}_{2}(i)$ for all $i \in I$. Since $v_{i}$ is continuous on $A \times \mathcal{M}^{\rho}(T \times A)$, we can appeal to Berge's maximum theorem to guarantee that $B(i, \cdot)$ is upper hemi-continuous. In particular, for any given $(i, \tau), B(i, \tau)$ is a closed set. Furthermore, for each $\tau \in \mathcal{M}^{\rho}(T \times A)$, since $v_{(\cdot)}(\cdot, \tau)$ is a measurable function on $I$, and a continuous function on $A$, we can apply [3, Theorem 18.19 and Theorem 18.13] to assert that there exists a measurable selection from the correspondence $B(\cdot, \tau)$. Let $\tilde{B}(i, \tau)=\{\alpha(i)\} \times B(i, \tau)$ for all $i \in I$ and for all $\tau \in \mathcal{M}^{\rho}(T \times A)$ where $\alpha(i)=\mathcal{G}_{1}(i)$ for all $i \in I$. It is easy to see that $\tilde{B}(i, \cdot)$ is also upper hemi-continuous on $\mathcal{M}^{\rho}(\mathcal{T} \times \mathcal{A})$ for each $i$ and $\tilde{B}(i, \tau)$ is closed-valued for any given $(i, \tau)$. Denote the correspondence $\tilde{B}(\cdot, \tau): I \longrightarrow T \times A$ by $\tilde{B}_{\tau}$. Now define a correspondence $\Phi: \mathcal{M}^{\rho}(T \times A) \longrightarrow \mathcal{M}^{\rho}(T \times A)$ by letting $\Phi(\tau)=\mathcal{D}_{\tilde{B}_{\tau}}$ where $\mathcal{D}_{\tilde{B}_{\tau}}=\left\{\lambda \tilde{f}^{-1}: \tilde{f}\right.$ is a measurable selection of $\left.\tilde{B}_{\tau}\right\}$. We now show that $\Phi$ is a nonempty, closed and convex valued, upper hemi-continuous correspondence from a non-empty convex compact subset of a locally convex space into itself. For any given $\tau \in \mathcal{M}^{\rho}(T \times A)$, there exists a measurable selection $f: I \longrightarrow A$ where $f(i) \in B(i, \tau)$. Let $h(i)=(\alpha(i), f(i))$ for all $i \in I$. It is clear that $h(i)$ is a measurable selection of $\tilde{B}_{\tau}$ and $\lambda h^{-1} \in \mathcal{M}^{\rho}(T \times A)$. Thus, $\Phi$ is nonempty-valued. If either (i) or (ii) is satisfied, we can apply Proposition C.P1 in the Appendix to assert that $\Phi(\tau)$ is also convex. Moreover, because $\tilde{B}_{\tau}$ is closed-valued, hence, compact-valued, and $\tilde{B}(i, \cdot)$ is upper hemi-continuous on $\mathcal{M}^{\rho}(T \times A)$, we can apply Proposition C.P2 and Proposition C.P3 in the Appendix respectively to assert that $\Phi$ is compact-valued and upper hemi-continuous. Thus, we can apply the Fan-Glicksberg fixed-point theorem to assert that there exists a $\tau^{*} \in \Phi\left(\tau^{*}\right)$, and thus, a measurable selection $f^{*}: I \longrightarrow A$ such that $\tau^{*}=\lambda\left(\alpha, f^{*}\right)^{-1}$. It is clear that $f^{*}$ is a Nash equilibrium.

Remark 1: It is clear that the procedure that relates the conventional form of a large game to the reformulated version studied here, and delineated at the end of Section 2, can be used to derive as straightforward corollaries the principal results of [21] and [12]. The interested reader can also check that the compactness of the action set $A$ can be relaxed with less restrictive assumptions that allow each player to choose her action from a compact subset of the complete countable metric space, as shown in [36].

Remark 2: Examples showing that a large game does not have a Nash equilibrium if the action set is uncountable are by now well-known; see [23] for references. These examples can be used to show that Theorem 1(i) is false if the countability hypothesis on $A$ is relaxed without any additional assumptions. What is more important in the context of this paper, of course, is that the result does not extend to the case where the trait space $T$ is uncountable even with a finite action set. Indeed, Example 1 discussed in the next section is based on an action set with only two elements!

Next, we turn to the necessity of saturated spaces when Nash equilibria exist in large games. The relevant benchmark result is due to [17, Theorem 4.6].

Proposition B. Let $(I, \mathcal{I}, \lambda)$ be an atomless probability space, and $A$ an uncountable compact metric space. Then $(I, \mathcal{I}, \lambda)$ is saturated if and only if every game $\mathcal{G}^{0}$ in terms of Definition 1 with player space $(I, \mathcal{I}, \lambda)$ and action space $A$ has a Nash equilibrium. ${ }^{27}$

We can now present our main result on the necessity of saturated spaces toward the existence of Nash equilibria in our reformulation of large games, namely, large games with traits.

\footnotetext{
${ }^{27}$ The proof of necessity part of this result in [17] is based on a local characterization of saturated spaces presented as their Corollary 4.4. An earlier unpublished version of [17] contained a more constructive proof of the necessity result. With the permission of the authors of [17], we provide this alternative proof as the proof of Lemma 1 so as to motivate the proof of Lemma 2 below. This lemma is essential in the proof of the necessity result in Theorem 2.
} 
Theorem 2. An atomless probability space $(I, \mathcal{I}, \lambda)$ is saturated if and only if every large game with traits $\mathcal{G}: I \longrightarrow T \times \mathcal{V}_{(A, T, \rho)}$ has a Nash equilibrium provided one of the following two conditions holds:

(i) A is uncountable,

(ii) $T$ is uncountable and $\rho$ is atomless.

Proof: Suppose that $(I, \mathcal{I}, \lambda)$ is saturated. For both (i) and (ii), by Theorem 1, there exists a Nash equilibrium.

We now prove the necessity parts. First, suppose that condition (i) holds but $(I, \mathcal{I}, \lambda)$ is not saturated. Thus, by Proposition $\mathrm{B}$, when $A$ is an uncountable compact metric space, there must exist a game in terms of Definition 1 with $(I, \mathcal{I}, \lambda)$ as the name space that does not have any Nash equilibrium. Let this game be $\mathcal{G}^{0}$. Thus, $\mathcal{G}^{0}$ is a measurable function from $I$ to $\mathcal{U}_{A}$ such that there does not exist any measurable function $f: I \longrightarrow A$ that satisfies for $\lambda$-almost all $i \in I, u_{i}\left(f(i), \lambda f^{-1}\right) \geq u_{i}\left(a, \lambda f^{-1}\right)$ for all $a \in A$, where $u_{i}=\mathcal{G}^{0}$.

Let $\mathcal{G}$ be a function from $I$ to $T \times \mathcal{V}_{(A, T, \rho)}$ such that for all $i \in I, \lambda \mathcal{G}_{1}^{-1}=\rho$ and $\mathcal{G}_{2}(i)=v_{i}$ where $v_{i}$ is defined as $v_{i}(a, \tau)=u_{i}\left(a, \tau_{A}\right)$ with $\tau_{A}$ being the marginal of $\tau$ on $A$ for any $a \in A$ and any $\tau \in \mathcal{V}_{(A, T, \rho)}$. It is easy to see that $\mathcal{G}$ is a game with traits that satisfies condition (i). Now suppose that any game with the structure described in Theorem 2 with condition (i) has a Nash equilibrium. Then there must exist a Nash equilibrium $f^{*}$ for this constructed game $\mathcal{G}$. That is, there is a measurable function $f^{*}: I \longrightarrow A$ such that for $\lambda$-almost all $i \in I$,

$$
v_{i}\left(f^{*}(i), \lambda\left(\mathcal{G}_{1}, f^{*}\right)^{-1}\right) \geq v_{i}\left(a, \lambda\left(\mathcal{G}_{1}, f^{*}\right)^{-1}\right) \text { for all } a \in A .
$$

Then, by the construction of the game $\mathcal{G}$, the measurable function $f^{*}$ also satisfies, for $\lambda$-almost all $i \in I$,

$$
u_{i}\left(f^{*}(i), \lambda f^{*-1}\right) \geq u_{i}\left(a, \lambda f^{*-1}\right) \text { for all } a \in A .
$$

This is a contradiction. Hence, $(I, \mathcal{I}, \lambda)$ must be saturated if condition (i) holds.

Now suppose condition (ii) holds but $(I, \mathcal{I}, \lambda)$ is not saturated. Suppose that every game with traits under condition (ii) has a Nash equilibrium. However, Lemma 3 in the Appendix shows that when $T$ is uncountable, $\rho$ is atomless, and $A=\{\bar{H}, \bar{T}\}$, there is a large game with traits $\mathcal{G}: I \longrightarrow T \times \mathcal{V}_{(A, T, \rho)}$ which does not have any Nash equilibrium. This is the contradiction that ensures that $(I, \mathcal{I}, \lambda)$ must be saturated as well if condition (ii) holds.

\section{A Dissonance: Four Illustrative Examples}

In this section we present three examples that point to a rather fundamental dissonance between discretized games with traits, and an idealized game modeled on the Lebesgue interval, and then use the fourth example to point a way towards a resolution based on saturated probability spaces.

In the classical example of a 2-player game of matching pennies, the players try to out-play each other; one player wants the two pennies to match, while the other does not. There is no pure-strategy Nash equilibrium for such a game. In this section, we first consider a large game of matching pennies in which the players with a multiplicity of traits are finely balanced. Such a game has no Nash equilibrium in pure-strategies. As already discussed in Remark 2, Theorem 1 does not extend to the general the trait space without assuming 
that the name space is saturated. What is interesting in Example 1 is that it shows the failure of the existence claim in Theorem 1 when the trait space $T$ is uncountable even when the action set is finite.

Example 1: We consider a large game that is a natural generalization of the matching pennies with two players. Let the space of players be the Lebesgue unit interval $(I, \mathcal{I}, \lambda)$ and the space of actions $A$ be the set $\{\bar{H}, \bar{T}\}$, representing Head and Tail respectively. Let the space of traits $T$ be the unit interval $I$. Assume that the players' traits are uniformly distributed on $I$; and thus let $\alpha$ be the identity mapping on $I$ and $\rho=\lambda$. We can interpret the trait of an individual players as normalized age (or complexion or income). For concreteness, let us use age.

For any $a \in A$ and $\tau \in \mathcal{M}^{\rho}(T \times A)$, let ${ }^{28} v_{i}(a, \tau)=-\int_{T \times A} 1_{[0, i) \times\{a\}}(t, x) d \tau$ for all $i \in I$, where $1_{C}$ is the indicator function of set $C$. Let $f$ be a strategy profile for the game, $\tau=\lambda(\alpha, f)^{-1}, \tau_{\bar{H}}([0, i))=$ $\lambda\left((\alpha, f)^{-1}([0, i) \times\{\bar{H}\})\right)$ and $\tau_{\bar{T}}([0, i))=\lambda\left((\alpha, f)^{-1}([0, i) \times\{\bar{T}\})\right)$. Namely, $\tau_{\bar{H}}([0, i))\left(\tau_{\bar{T}}([0, i))\right)$ is the proportion of all players younger than $i$ who play Heads (Tails). It is easy to see that $v_{i}(\bar{H}, \tau)=-\tau_{\bar{H}}([0, i))$ and $v_{i}(\bar{T}, \tau)=-\tau_{\bar{T}}([0, i))$. Hence, player $i$ 's optimal response is respectively Tail if $\tau_{\bar{H}}([0, i))>\tau_{\bar{T}}([0, i))$, Head if $\tau_{\bar{T}}([0, i))>\tau_{\bar{H}}([0, i))$, and Head or Tail if $\tau_{\bar{H}}([0, i))=\tau_{\bar{T}}([0, i))$. It means that the $i^{\text {th }}$ player always tries to balance out those players younger than her. If there are more younger players who play Heads (Tails), then player $i$ will play Tail (Head).

We now have to show that these specifications do indeed give us a large game with traits as defined in Definition 2, and that this game has no (pure-strategy) Nash equilibrium. This is a rather technical argument: we simply report it as a claim, prove it formally in the Appendix, and satisfy ourselves here with an intuitive sketch of the argument.

Claim 1. Let $\mathcal{G}$ be a function such that for all $i \in I, \mathcal{G}(i)=\left(\alpha(i), v_{i}\right)$, where $\alpha(i)$ and $v_{i}$ are specified as above. Then $(i) \mathcal{G}$ is a measurable function from $I$ to $T \times \mathcal{V}_{(A, T, \rho)}$, a game which satisfies all the requirements of Definition 2, and (ii) there does not exist a Nash equilibrium for $\mathcal{G}$.

In terms of the basic intuition for the non-existence of equilibrium for this game with traits, suppose to the contrary that $f^{*}$ is an equilibrium in pure strategies. Then for every $i \in I$, the respective sets of younger players who play Heads and Tails must have the same measure. Let $F=f^{*-1}(\{\bar{H}\})$ be the set of all the players who play Heads. Then the measurable set $F$ cuts through $[0, i)$ into half for every $i \in I$, which means that $F$ has probability $1 / 2$ and is stochastically independent of all the intervals $[0, i$ ) (and thus all the measurable sets). This is impossible.

Next, we turn to a second example of a sequence of finite games which draw on the intuition of the large matching-penny game in Example 1, but for which somewhat surprisingly there does exist an exact Nash equilibrium irrespective of the number of players.

Example 2: Fix a positive integer $n$ and let $I_{n}=\left\{k / 2^{n}: k=0,1, \ldots, 2^{n}\right\}$. Let $\lambda_{n}$ denote the counting measure on $I_{n}$, i.e., $\lambda_{n}(i)=1 /\left(2^{n}+1\right)$ for every $i \in I_{n}$. Let $A=\{\bar{H}, \bar{T}\}$ be the set of actions. Let $T_{n}=I_{n}, \alpha_{n}: I_{n} \longrightarrow T_{n}$ be $\alpha_{n}(i)=i$ and $\rho_{n}=\lambda_{n} \alpha_{n}^{-1}=\lambda_{n}$. For any $a \in A$ and $\tau_{n} \in \mathcal{M}^{\rho_{n}}\left(T_{n} \times A\right)$, let $v_{i}^{n}\left(a, \tau_{n}\right)=-\int_{T_{n} \times A} 1_{[0, i) \times\{a\}}(t, x) d \tau_{n}$. Notice that when $i=0, v_{i}(\cdot, \cdot)$ is identically zero. Define

\footnotetext{
${ }^{28}$ This payoff function is adapted from the example in Remark 3 of [32], where the large game is considered in the setting of no-traits. As discussed in the introduction, the distinction between player-names and traits is conceptually important for the result in this paper.
} 
$\mathcal{G}_{n}: I_{n} \longrightarrow T_{n} \times \mathcal{V}_{\left(A, T_{n}, \rho_{n}\right)}$ as $\mathcal{G}_{n}(i)=\left(\alpha_{n}(i), v_{i}^{n}\right)$ for all $i \in I_{n}$. Given any $a \in A$ and any measurable function $f_{n}$ from $I_{n}$ to $A$ where $f_{n}(i)=a$,

$$
v_{i}^{n}\left(a, \lambda_{n}\left(\alpha_{n}, f_{n}\right)^{-1}\right)=-\int_{T_{n} \times A} 1_{[0, i) \times\{a\}}(t, x) d \lambda_{n}\left(\alpha_{n}, f_{n}\right)^{-1}=-\lambda_{n}\left([0, i) \cap f_{n}^{-1}(a)\right) .
$$

Let $f_{n}^{*}: I_{n} \longrightarrow A$ be $f_{n}^{*}\left(k / 2^{n}\right)=\bar{H}$ when $k$ is even, and $f_{n}^{*}\left(k / 2^{n}\right)=\bar{T}$ otherwise. We will show that $f_{n}^{*}$ is a Nash equilibrium of $\mathcal{G}_{n}$. Player 0 is always indifferent between $\bar{H}$ and $\bar{T}$. So, $f_{n}^{*}(0)=\bar{H}$ is a best response for player 0. For player $i=k / 2^{n}$ where $k$ is positive and even, $\lambda_{n}\left([0, i) \cap f_{n}^{*-1}(\bar{H})\right)=\lambda_{n}\left([0, i) \cap f_{n}^{*-1}(\bar{T})\right)$. So, $f_{n}^{*}(i)=\bar{H}$ is a best response for $i$. For player $i=k / 2^{n}$ where $k$ is odd, $\lambda_{n}\left([0, i) \cap f_{n}^{-1}(\bar{H})\right)>$ $\lambda_{n}\left([0, i) \cap f_{n}^{-1}(\bar{T})\right)$. So, $\bar{T}$ is the best response for $i$. Hence, $f_{n}^{*}$ is a Nash equilibrium of $\mathcal{G}_{n}$. This is true for any integer $n$.

Undoubtedly useful as the above example is, it suffers from the fact that the discretized version of the game $\mathcal{G}$ in Example 1 in the form of the sequence of games does not converge to the idealized limit game $\mathcal{G}$. However, we draw on the basic intuition behind Example 2 to present another example that remedies this deficiency - it is a clear demonstration of the violation of the upper hemi-continuity of equilibrium correspondence defined on this common name-space.

Example 3: We follow the notation in Example 1. Fix a positive integer $n$, and for any $a \in A$ and $\tau \in \mathcal{M}^{\rho}(T \times A)$, let

$$
\bar{v}_{i}^{n}(a, \tau)= \begin{cases}v_{1}(a, \tau) & \text { for } i=1 \\ v_{k / 2^{n}}(a, \tau) & \text { for } i \in\left[\frac{k}{2^{n}}, \frac{k+1}{2^{n}}\right) \text { where } k=0,1, \ldots, 2^{n}-1 .\end{cases}
$$

Let $\overline{\mathcal{G}}_{n}(i)=\left(\alpha(i), \bar{v}_{i}^{n}\right)$ for all $i \in I$. It is clear that $\overline{\mathcal{G}}_{n}$ is a measurable function from $I$ to $T \times \mathcal{V}_{(A, T, \rho)}$. Hence, $\overline{\mathcal{G}}_{n}$ is a large game with traits.

Let $\bar{f}_{n}: I \longrightarrow A$ be a function such that $\bar{f}_{n}(i)$ is $\bar{H}$ when $i=1$ or $i \in\left[\frac{k}{2^{n}}, \frac{k+1}{2^{n}}\right)$ where $k$ is even between 0 and $2^{n}-1$, and equals $\bar{T}$ otherwise. We now show that $\bar{f}_{n}$ is a Nash equilibrium of $\overline{\mathcal{G}}_{n}$. For any $a \in A$ and $i \in\left[\frac{k}{2^{n}}, \frac{k+1}{2^{n}}\right)$ where $k$ is a integer between 0 and $2^{n}-1$, it is clear that $\bar{v}_{i}^{n}\left(a, \lambda\left(\alpha, \bar{f}_{n}\right)^{-1}\right)=$ $-\lambda\left(\left[0, \frac{k}{2^{n}}\right) \cap \bar{f}_{n}^{-1}(a)\right)$. Thus, for $i \in\left[0, \frac{1}{2^{n}}\right), \bar{v}_{i}^{n}=v_{0}=0$, and hence $\bar{f}_{n}(i)=\bar{H}$ is a best response for player $i$. For $i \in\left[\frac{1}{2^{n}}, \frac{2}{2^{n}}\right), \bar{v}_{i}^{n}\left(\bar{H}, \lambda\left(\alpha, \bar{f}_{n}\right)^{-1}\right)=-\frac{1}{2^{n}}$ while $\bar{v}_{i}^{n}\left(\bar{T}, \lambda\left(\alpha, \bar{f}_{n}\right)^{-1}\right)=0$. This is to say, $\bar{f}_{n}(i)=\bar{T}$ is a best response for $i \in\left[\frac{1}{2^{n}}, \frac{2}{2^{n}}\right)$. Similarly, we can obtain that $\bar{f}_{n}$ is a best response for each $i \in I$. Therefore, $\bar{f}_{n}$ is a Nash equilibrium of $\overline{\mathcal{G}}_{n}$. This is true for any integer $n$.

By the proof of Claim 1 in the Appendix, $\left|v_{i}(\cdot, \cdot)-v_{j}(\cdot, \cdot)\right| \leq|j-i|$ for any $i$ and $j$ in $I$. Hence, $\left|\bar{v}_{i}^{n}(\cdot, \cdot)-v_{j}(\cdot, \cdot)\right| \leq \frac{1}{2^{n}}$ for each $i \in I$. Therefore, $\left\{\bar{v}^{n}\right\}$ converges to $v$ uniformly as functions in $\mathcal{V}_{(A, T, \rho)}$. Thus, it is clear that the sequence $\left\{\overline{\mathcal{G}}_{n}\right\}$ above converges weakly to the game $\mathcal{G}$ in Example 1 in distribution. However, each $\overline{\mathcal{G}}_{n}$ in the sequence has a Nash equilibrium while the limit game $\mathcal{G}$ does not. Observe that the sequence $\left\{\bar{f}_{n}\right\}$ does not converge almost everywhere but only in distribution whereby $\left\{\lambda\left(\bar{f}_{n}\right)^{-1}\right\}$ converges weakly to the uniform distribution $\varsigma^{*}$ on $A$. However, there does not exist a Nash equilibrium $f^{*}$ of $\mathcal{G}$ such that $\lambda\left(f^{*}\right)^{-1}=\varsigma^{*}$.

We are now ready to address the dissonance between the limiting and idealized limit cases, for both the existence and upper hemi-continuity of Nash equilibrium, as brought out in the above examples, by 
considering an example modeled on a saturated probability space as an extension of the Lebesgue unit interval.

Example 4: Consider a new game $\mathcal{G}^{\prime}$ in which everything is the same as the game $\mathcal{G}$ in Example 1 except that the player space, as formalized by the Lebesgue unit interval $(I, \mathcal{I}, \lambda)$ in Example 1, is now replaced by a saturated extension $(I, \mathcal{S}, \eta) .{ }^{29}$ Clearly, $\left\{\overline{\mathcal{G}}_{n}\right\}$ in Example 3 also converges weakly to $\mathcal{G}^{\prime}$ in distribution. By Theorem 1, $\mathcal{G}^{\prime}$ has a Nash equilibrium. Following [24], we can obtain an explicit expression for a Nash equilibrium $f^{*}$ of $\mathcal{G}^{\prime}$ with $\eta\left(f^{*}\right)^{-1}=\varsigma^{*}$.

Towards this end, we follow the construction in [35]. Let $K=[0,1]$ and $(K, \mathcal{K}, \kappa)$ be a probability space obtained from a Loeb probability space via a bijection. Consider a subset $C$ of $I \times K$. An appeal to [35, Lemma 3] allows us to assert that there exists a disjoint family $\mathcal{C}=\left\{C_{k}: k \in K=[0,1]\right\}$ of subsets of $I$ such that $\bigcup_{k \in K} C_{k}=I$, and for each $k \in K, \lambda_{*}\left(C_{k}\right)=0$ and $\lambda^{*}\left(C_{k}\right)=1$, where $\lambda_{*}$ and $\lambda^{*}$ are the respective inner and outer measures of the Lebesgue measure $\lambda$. Let $C$ be $\left\{(i, k) \in I \times K: i \in C_{k}, k \in K\right\}, K_{1}$ be a $\mathcal{K}$-measurable subset of $K$ with $\kappa\left(K_{1}\right)=1 / 2$, and $K_{2}=K-K_{1}$. Let $S_{1}=\bigcup_{k \in K_{1}} C_{k}$ and $S_{2}=\bigcup_{k \in K_{2}} C_{k}$, where $C_{k} \in \mathcal{C}$.

Recall that $\mathcal{C}$ is a partition of $I$, and that therefore $S_{1} \cap S_{2}=\emptyset, S_{1} \cup S_{2}=I$. Now, one can check that the following result holds, along the lines of the proof of [24, Lemma 2].

Proposition C. For $j=1,2, S_{j} \in \mathcal{S}$, but $S_{j} \notin \mathcal{I}$. Moreover, $\eta\left(S_{j} \cap[0, i)\right)=i / 2$, for any $i \in[0,1]$, and in particular, $\eta\left(S_{j}\right)=1 / 2$.

Now, let $f^{*}$ be a function from $I$ to $\{\bar{H}, \bar{T}\}$ as follows,

$$
f^{*}(i)= \begin{cases}\bar{H}, & \text { if } i \in S_{1} \\ \bar{T}, & \text { if } i \in S_{2}\end{cases}
$$

Clearly, $f^{*}$ is $\mathcal{S}$-measurable. Let $\tau^{\prime}=\eta\left(\alpha, f^{*}\right)^{-1}$. By Proposition $\mathrm{C}$, for any $i \in[0,1], \tau_{\bar{H}}^{\prime}([0, i))=$ $\eta\left[\left(\alpha, f^{*}\right)^{-1}([0, i) \times\{\bar{H}\})\right]=\eta\left([0, i) \cap S_{1}\right)=i / 2$, and similarly, $\tau_{\bar{T}}^{\prime}([0, i))=\eta\left[\left(\alpha, f^{*}\right)^{-1}([0, i) \times\{\bar{T}\})\right]=$ $\eta\left([0, i) \cap S_{2}\right)=i / 2$. Hence, for each player $i \in I, v_{i}\left(\bar{H}, \tau^{\prime}\right)=-\tau_{\bar{H}}^{\prime}([0, i))=-\tau_{\bar{T}}^{\prime}([0, i))=v_{i}\left(\bar{T}, \tau^{\prime}\right)$. In other words, In other words, player $i$ 's action $f^{*}(i)$, which is either $\bar{H}$ or $\bar{T}$, is the best response against $\tau^{\prime}$. Therefore $f^{*}$ is a Nash equilibrium of this new game game, one for which, it is easy to check, $\eta\left(f^{*}\right)^{-1}=\varsigma^{*}$. This is to say, the sequence of Nash equilibria $\left\{\bar{f}_{n}\right\}$ in Example 3 converges weakly to this $f^{*}$ in $\mathcal{M}(A)$.

Then, the dissonance between the limiting and idealized limit cases for both the existence and upper hemi-continuity of Nash equilibrium is resolved in this example.

We conclude this section with a remark that bears on the necessity of saturation in Example $4 .{ }^{30}$

Remark 3: Instead of using $(I, \mathcal{S}, \eta)$ as the player-space in Example 4, one can show the existence of a Nash equilibrium simply by working with a countably-generated Lebesgue extension, as argued in [24]. However, just as in that paper, and in the light of Theorem 4 below, there will exist another example of a game with traits that would require yet another extension. In fact, let $\varphi$ be the mapping from $(I, \mathcal{S}, \eta)$ to the product space $[0,1] \times A$ with the usual product Borel $\sigma$-algebra such that $\varphi(i)=\left(i, f^{*}(i)\right)$, where $f^{*}$ is the Nash

\footnotetext{
${ }^{29}$ The possibility of such an extension has been shown in [16], [27] and [35]. Note that the issue in [35] is the construction of an additional structure of rich Fubini extensions associated with a Lebesgue extension.

${ }^{30}$ Given the technical nature of this remark, the reader may choose to skip it on a first reading.
} 
equilibrium in Example 4. Let $\sigma(\varphi)$ be the $\sigma$-algebra generated by $\varphi$, and $\theta$ be the restriction of $\eta$ to $\sigma(\varphi)$. Hence, $(I, \sigma(\varphi), \theta)$ is a countably-generated sub- $\sigma$-algebra of $\mathcal{S}$ that extends the Lebesgue unit interval and allows the equilibrium $f^{*}$ to be measurable. Thus, one can construct a new game with traits by replacing the name space of the game $\mathcal{G}$ in Example 1 by $(I, \sigma(\varphi), \theta)$ to resolve the dissonance of the first three examples.

\section{Saturated Probability Spaces and Closed-Graph Theorems}

We now build on the previous Section 4 to address the upper hemi-continuity of the Nash equilibrium correspondence for a large game with traits. Example 4 shows that if the player space of the game in Example 1 is replaced by its saturated extension, then there exists a Nash equilibrium in the modified game. Moreover, the distribution induced by this Nash equilibrium is the limit of the distributions induced by Nash equilibria of the sequence of games in Example 3, a sequence that sets up the basic intuition behind Example 2 into a common name-space represented by the Lebesgue interval. Furthermore, Example 1, 3 and 4 all share the same trait space, and it is the fact that Example 4, and not Example 1, has the requisite properties that prompts the need to look into necessity considerations. As Theorem 4 testifies, these hopes are amply fulfilled.

The basic idea of the closed-graph property of the Nash equilibrium correspondence is clear enough: it simply asserts that any sequence of Nash equilibria of a sequence of games converging to a limit game, converge to a Nash equilibrium of the limit game. All that needs to be made precise is the sequence of games that is considered and the mode of convergence that is adopted. In keeping with the development so far, there is little choice as regards the latter but to work with convergence in distribution. However, as regards the former, one can consider two different settings: one where the distribution of traits in the sequence of games is allowed to vary, and one where it is not. We present two results, and whereas the first can be seen as the more general in terms of providing a sufficiency result for the closed-graph property, it is the second which furnishes the if-and-only-if result as far as the saturation property is concerned.

Let $\left\{\left(I^{n}, \lambda^{n}\right)\right\}$ be a sequence of probability spaces where $\sup _{i \in I^{n}} \lambda^{n}(i) \rightarrow 0$. To allow the possibility that a sequence of games has different distributions of traits, we need to work with $\mathcal{M}(T \times A)$, the space of Borel probability measures on $T \times A$ rather than $\mathcal{M}^{\rho}(T \times A), \rho$ being a fixed distribution of traits. However, given the generality of the space of traits $T$ that we have been working with, we are no longer guaranteed the compactness of the space $\mathcal{M}(T \times A)$. Hence, for our first result, Theorem 3 below, we assume that $T$ is compact. We can now endow $\mathcal{V}$, the space of all continuous functions on the product space $A \times \mathcal{M}(T \times A)$, with its sup-norm topology and the resulting Borel $\sigma$-algebra. ${ }^{31}$ For each $n \in \mathbb{N}$, let a game with traits $\mathcal{G}^{n}$ be a mapping from $I^{n}$ into $T \times \mathcal{V}$ with $\alpha^{n}(i) \equiv \mathcal{G}_{1}^{n}(i) \in T$ and $v_{i}^{n} \equiv \mathcal{G}_{2}^{n}(i) \in \mathcal{V}$ for each $i \in I^{n}$. A mapping $f^{n}: I^{n} \longrightarrow A$ is a Nash equilibrium of $\mathcal{G}^{n}$, if for $\lambda^{n}$-almost all $i \in I^{n}$,

$$
v_{i}^{n}\left(f^{n}(i), \lambda^{n}\left(\alpha^{n}, f^{n}\right)^{-1}\right) \geq v_{i}^{n}\left(a, \lambda^{n}\left(\alpha^{n},\left(a, f_{-i}^{n}\right)\right)^{-1}\right) \text { for all } a \in A,
$$

where $^{32}\left(a, f_{-i}^{n}\right): I^{n} \longrightarrow A$ is a mapping defined by $\left(a, f_{-i}^{n}\right)(j)=a$ if $j=i$ and $\left(a, f_{-i}^{n}\right)(j)=f^{n}(j)$

\footnotetext{
${ }^{31}$ The reader may wish to review the basic notation developed in paragraph 2 of Section 2 above, and be clear on the difference between $\mathcal{V}$ and $\mathcal{V}_{(A, T, \rho)}$.

${ }^{32}$ Note that when $\lambda^{n}$ is atomless, this is equivalent to $v_{i}^{n}\left(f^{n}(i), \lambda^{n}\left(\alpha^{n}, f^{n}\right)^{-1}\right) \geq v_{i}^{n}\left(a, \lambda^{n}\left(\alpha^{n}, f^{n}\right)^{-1}\right)$. Thus, this general inequality covers both the definition of Nash equilibrium as in Example 2 as well as the one in Definition 2.
} 
otherwise.

We can now present a basic formalization of the closed graph property.

Definition 4. The Nash equilibrium correspondence of a large game with traits $\mathcal{G}: I \longrightarrow T \times \mathcal{V}$ has the closed graph property if for any sequence of games with traits $\left\{\mathcal{G}^{n}\right\}$ which converges weakly ${ }^{33}$ to $\mathcal{G}$, and the sequence of Nash equilibria $f^{n}$ of $\mathcal{G}^{n}$ for each $n \in \mathbb{N}$ is such that $\lambda^{n}\left\{\left(f^{n}\right)^{-1}\right\}$ converges weakly to some $\varsigma \in \mathcal{M}(A)$, then there exists a Nash equilibrium $f$ of $\mathcal{G}$ such that $\lambda f^{-1}=\varsigma$.

Motivated by the examples in Section 4, we have the following general result concerning the closed graph property of a large game with traits, one in which there is no requirement that the distribution of traits be fixed as we move along the considered sequence of games.

Theorem 3. If $(I, \mathcal{I}, \lambda)$ is a saturated probability space, then the Nash equilibrium correspondence of a large game with traits $\mathcal{G}: I \longrightarrow T \times \mathcal{V}$ has the closed graph property. ${ }^{34}$

Proof: Let $f^{n}$ be a Nash equilibrium of $\mathcal{G}^{n}$ for each $n \in \mathbb{N}$ such that $\left\{\lambda^{n}\left(f^{n}\right)^{-1}\right\}$ converges weakly to some $\varsigma$. Let $\mu^{n}=\lambda^{n}\left(\mathcal{G}^{n}, f^{n}\right)^{-1}$ for all $n \in \mathbb{N}$. Since $\left\{\lambda^{n}\left(\mathcal{G}^{n}\right)^{-1}\right\}$ converges weakly to $\lambda \mathcal{G}^{-1}$, by [17, Lemma 2.1] we know that some subsequence of $\left\{\mu^{n}\right\}$ converges weakly to $\mu \in \mathcal{M}(T \times \mathcal{V} \times A)$ whose marginals on $T \times \mathcal{V}$ and $A$ are $\lambda \mathcal{G}^{-1}$ and $\varsigma$ respectively. Without loss of generality, let the subsequence be the sequence itself. Because $(I, \mathcal{I}, \lambda)$ is saturated, Proposition A implies that there exists a measurable function $f$ such that $\lambda(\mathcal{G}, f)^{-1}=\mu$. It is clear that $\lambda f^{-1}=\varsigma$. Thus, in order to show that $\mathcal{G}$ has the closed graph property of its Nash equilibrium correspondence, it is sufficient to show that this measurable function $f$ is a Nash equilibrium of $\mathcal{G}$.

Towards this end, let $\tau$ be the marginal of $\mu$ on $T \times A$ and $\tau^{n}$ be the marginal of $\mu^{n}$ on $T \times A$ for each $n$. Since $A$ is separable it has a countable dense subset $A_{0}$. Fix any $a \in A_{0}$. For all $n \in \mathbb{N}$, we have for $\lambda_{n}$-almost all $i \in I_{n}$,

$$
v_{i}^{n}\left(f^{n}(i), \tau^{n}\right) \geq v_{i}^{n}\left(a, \lambda^{n}\left(\alpha^{n},\left(a, f_{-i}^{n}\right)\right)^{-1}\right) .
$$

Let $\tau_{a}^{n}=\lambda^{n}\left(\alpha^{n},\left(a, f_{-i}^{n}\right)\right)^{-1}, v^{n}=\mathcal{G}_{2}$ for all $n \in \mathbb{N}$, and $v=\mathcal{G}_{2}$. Because $\left\{\mu^{n}\right\}$ converges weakly to $\mu$, we know that $\left\{\tau^{n}\right\}$ converge weakly to $\tau,\left\{v_{n}\right\}$ converges weakly to $v$ and $\left\{f^{n}\right\}$ converges weakly to $f$.

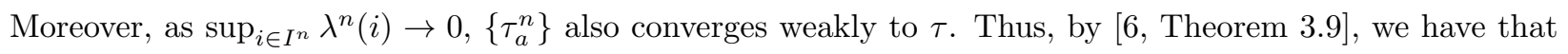
$\lambda^{n}\left(v^{n}, f^{n}, \tau^{n}, \tau_{a}^{n}\right)^{-1}$ converges weakly to $\lambda(v, f, \tau, \tau)^{-1}$ in $\mathcal{M}(\mathcal{V} \times A \times \mathcal{M}(T \times A) \times \mathcal{M}(T \times A))$. Now let $\psi\left(\tilde{v}, \tilde{a}, \tilde{\tau}, \tilde{\tau}_{a}\right)=\tilde{v}(\tilde{a}, \tilde{\tau})-\tilde{v}\left(a, \tilde{\tau}_{a}\right)$ for any $\tilde{v} \in \mathcal{V}, \tilde{a} \in A$, and $\tilde{\tau}, \tilde{\tau}_{a} \in \mathcal{M}(T \times A)$. It is clear that $\psi$ is a bounded continuous function on $\mathcal{V} \times A \times \mathcal{M}(T \times A) \times \mathcal{M}(T \times A)$. Let $h_{n}(i)=\psi\left(v_{i}^{n}, f^{n}(i), \tau^{n}, \tau_{a}^{n}\right)$ for all $n \in \mathbb{N}$ and $h(i)=\psi\left(v_{i}, f(i), \tau, \tau\right)$. It follows that $\left\{h_{n}(i)\right\}$ converges weakly to $h(i)$. As $\lambda^{n}\left(h_{n}\right)^{-1}([0, \infty))=1$ for each $n \in \mathbb{N}$, we have $\lambda h^{-1}([0, \infty)) \geq \lim \sup _{n \rightarrow \infty} \lambda^{n}\left(h_{n}\right)^{-1}([0, \infty))=1$. Hence, for $\lambda$-almost all $i$,

$$
v_{i}(f(i), \tau) \geq v_{i}(a, \tau)
$$

\footnotetext{
${ }^{33}$ This simply means that $\left\{\mathcal{G}^{n}\right\}$ converges weakly to $\mathcal{G}$ in distribution; also see Footnote 21 in this connection. To re-emphasize, $\left\{\lambda^{n}\left(\mathcal{G}^{n}\right)^{-1}\right\}$ converges weakly to $\lambda \mathcal{G}^{-1}$ in $\mathcal{M}(T \times \mathcal{V})$.

${ }^{34}$ For equilibrium distributions, as considered in [26], the closed graph property is a standard result in the earlier literature; see, for example, [18, Theorem 3.2] and [29, Theorem 2]. What is interesting is that such a property fails in the usual Nash equilibrium setting. The idea of the proof given below follows from that of Theorem 4.7 in [17].
} 
Finally, by grouping countably many null sets together, we see that for $\lambda$-almost all $i \in I$,

$$
v_{i}(f(i), \tau) \geq v_{i}(a, \tau), \text { for all } a \in A,
$$

which implies that this $f$ is a Nash equilibrium of $\mathcal{G}$. The proof is now complete.

Next, we turn to the situation when the distribution of traits is kept fixed along the considered sequence of games. This requires us to work with $\mathcal{V}_{(A, T, \rho)}$ instead of $\mathcal{V}$, and present the corresponding modification of the definition for the closed graph property.

Definition 5. The Nash equilibrium correspondence of a large game with traits $\mathcal{G}: I \longrightarrow T \times \mathcal{V}_{(A, T, \rho)}$ has the closed graph property if for any sequence of games $\left\{\mathcal{G}^{n}\right\}$ with a given distribution of traits ${ }^{35}$ which converges weakly to $\mathcal{G}$, and the sequence of Nash equilibria $f^{n}$ of $\mathcal{G}^{n}$ is such that $\left\{\lambda^{n}\left(f^{n}\right)^{-1}\right\}$ converges weakly to some $\varsigma \in \mathcal{M}(A)$, then there exists a Nash equilibrium $f$ of $\mathcal{G}$ such that $\lambda f^{-1}=\varsigma$.

The real surprise, of course, is perhaps the next result which shows that saturation of the name-space, unlike Theorem 3, is not only sufficient but also necessary for the closed graph property once it is reformulated along the lines of Definition 5.

Theorem 4. $(I, \mathcal{I}, \lambda)$ is saturated if and only if every large game with traits $\mathcal{G}: I \longrightarrow T \times \mathcal{V}_{(A, T, \rho)}$ has the closed graph property of its Nash equilibrium correspondence, provided one of the following two conditions holds:

(i) A is uncountable,

(ii) $T$ is uncountable and $\rho$ is atomless.

Proof: For the sufficiency result, one can check every step in the proof of Theorem 3 follows if $\mathcal{V}$ there is replaced by $\mathcal{V}_{(A, T, \rho)}$. Thus, we only need to prove the necessity part of the result. Towards this end, suppose $(I, \mathcal{I}, \lambda)$ is not saturated. When either (i) or (ii) holds, it follows from Theorem 2 there exists a large game with traits $\mathcal{G}: I \longrightarrow T \times \mathcal{V}_{(A, T, \rho)}$ which has no Nash equilibrium. Let $\mu=\lambda \mathcal{G}^{-1}$. Fix a saturated probability space $(\Omega, \mathcal{A}, P)$. Since $(\Omega, \mathcal{A}, P)$ is atomless, there exists a large game with traits $\mathcal{G}^{\prime}:(\Omega, \mathcal{A}, P) \longrightarrow T \times \mathcal{V}_{(A, T, \rho)}$ such that $\mu=P\left(\mathcal{G}^{\prime}\right)^{-1}$. It then follows from Theorem 1 that there exists a Nash equilibrium $f^{\prime}$ of $\mathcal{G}^{\prime}$. Now consider a sequence of games with traits $\left\{\mathcal{G}^{n}\right\}$ where $\mathcal{G}^{n}=\mathcal{G}^{\prime}$ for all $n \in \mathbb{N}$. Let $f^{n}=f^{\prime}$ for all $n \in \mathbb{N}$. It is clear that $\left\{\mathcal{G}^{n}\right\}$ converges weakly to $\mathcal{G}$ and $\left\{f^{n}\right\}$ converges weakly to $f^{\prime}$. Hence, Nash equilibrium correspondence of $\mathcal{G}$ does not have the closed graph property, giving us the contradiction that we seek.

\section{Approximations and Asymptotic Implementation}

Theorems 1 to 4 concern an idealized limit game based on a saturated probability space interpreted as a space of player's names, and the two results presented in this section round-off the theory by bringing in finite games, and approximate Nash equilibria of such games, into active play. However, the two results that we present give the section a split identity. The first stays with the idealized game, and asks how hypotheses

\footnotetext{
${ }^{35}$ Again, to re-emphasize, each member $\mathcal{G}^{n}$ of the sequence shares the same trait specification $(T, \rho)$.
} 
pertaining to a sequence of finite games converging to it bear on its set of Nash equilibrium. This result has interesting implications for upper hemi-continuity, and can be seen as a supplement to Theorem 3 . The second result takes leave of the continuum and presents a result on the existence of an approximate Nash equilibrium for a large but finite game. It is by now a requirement of the field that results on idealized games, or economies for that matter, be translated into large but finite settings to ensure that they are not simply artifacts of the way the continuum has been modeled. Indeed, the examples of Section 3 are also directed towards this end, though somewhat obliquely. However, it is worth emphasizing, without claiming too much in the other direction, that an added methodological aspect has to be introduced for obtaining such results, one that relies on the fact that Loeb spaces, and Loeb counting spaces in particular, are saturated. ${ }^{36}$

Let $I^{n}$ be the set of the first $n$ positive integers and with the counting probability measure $\lambda^{n}$ on its power set $\mathcal{I}^{n}$ and $\mathcal{V}$ the space of all continuous functions on the product space $A \times \mathcal{M}(T \times A)$ based on its sup-norm topology and endowed with its resulting Borel $\sigma$-algebra. Let $T$ be compact. For each $n \geq 1$, let a finite game with traits $\mathcal{G}^{n}$ be a mapping from $I^{n}$ into $T \times \mathcal{V}$ with $\alpha^{n}(i) \equiv \mathcal{G}_{1}^{n}(i)$ and $v_{i}^{n} \equiv \mathcal{G}_{2}^{n}(i)$ for each $i \in I^{n}$.

Definition 6. For any $\epsilon>0$, a mapping $f^{n}: I^{n} \longrightarrow A$ is an $\epsilon$-approximate equilibrium of $\mathcal{G}^{n}$, if for all $i \in I_{\epsilon}^{n}$ where $I_{\epsilon}^{n} \subseteq I_{n}$ with $\lambda^{n}\left(I_{\epsilon}^{n}\right)>1-\epsilon$,

$$
v_{i}^{n}\left(f^{n}(i), \lambda^{n}\left(\alpha^{n}, f^{n}\right)^{-1}\right) \geq v_{i}^{n}\left(a, \lambda^{n}\left(\alpha^{n}, f^{n}\right)^{-1}\right)-\epsilon, \text { for all } a \in A .
$$

We now present an approximate version of Theorem $3 .^{37}$

Proposition 1. If $(I, \mathcal{I}, \lambda)$ is a saturated probability space, then for any large game with traits $\mathcal{G}: I \longrightarrow T \times \mathcal{V}$, suppose that there exists a sequence of finite games $\left\{\mathcal{G}^{n}\right\}$ which converges weakly to $\mathcal{G}$, such that, there exists an $\epsilon^{n}$-approximate equilibrium $f^{n}$ of $\mathcal{G}^{n}$ where $\left\{\epsilon^{n}\right\}$ converges to 0 and $\left\{\lambda^{n}\left(f^{n}\right)^{-1}\right\}$ converges weakly to some $\varsigma \in \mathcal{M}(A)$, then there exists a Nash equilibrium $f$ of $\mathcal{G}$ such that $\lambda f^{-1}=\varsigma$.

Proof: The proof modifies the proof of Theorem 3 above by inserting the epsilons in the "right" places. Use identical notation as in that proof so as to show that the measurable function $f$ is a Nash equilibrium of $\mathcal{G}$. Then, by [6, Theorem 3.9], $\lambda^{n}\left(v^{n}, f^{n}, \tau^{n}\right)^{-1}$ converges weakly to $\lambda(v, f, \tau)^{-1}$ in $\mathcal{M}(\mathcal{V} \times A \times \mathcal{M}(T \times A))$. Fix $a \in A_{0}$. It follows that $h_{n}(i)=v_{i}^{n}\left(f^{n}(i), \tau^{n}\right)-v_{i}^{n}\left(a, \tau^{n}\right)$ converges weakly to $h(i)=v_{i}(f(i), \tau)-v_{i}(a, \tau)$. By [6, Theorem 3.1], $h_{n}(i)+\epsilon^{n}$ also converges weakly to $h(i)$. Moreover, since $\lambda^{n}\left(h_{n}+\epsilon^{n}\right)^{-1}([0, \infty))>1-\epsilon^{n}$ for each $n$, we have $\lambda h^{-1}([0, \infty)) \geq \limsup _{n \rightarrow \infty} \lambda^{n}\left(h_{n}+\epsilon^{n}\right)^{-1}([0, \infty))=1$ as $\left\{\epsilon_{n}\right\}$ converges to 0 . Hence, for $\lambda$-almost all $i \in I, v_{i}(f(i), \tau) \geq v_{i}(a, \tau)$. Thus, by arguments similar to the last paragraph in the proof of Theorem 3, it is clear that $f$ is a Nash equilibrium of $\mathcal{G}$.

For the next result, we shall need the following definition.

Definition 7. Let $\left\{g_{n}\right\}_{n \in \mathbb{N}}$ be a sequence of measurable mappings from a probability space $\left(Y, \mathcal{Y}, \mu_{Y}\right)$ to a Polish space $X$ equipped with its Borel $\sigma$-algebra $\mathcal{B}(X)$. It is said to be tight if for any $\epsilon>0$, there exists a compact subset $K_{\epsilon}$ of $X$ such that for all $n \in \mathbb{N}, \mu_{Y}\left(g_{n}^{-1}\left(K_{\epsilon}\right)\right)>1-\epsilon$.

\footnotetext{
${ }^{36}$ In the earlier papers of Brown, Robinson, Khan and Rashid, as comprehensively surveyed in Anderson [4], the results of the idealized model were derived $a b$ initio, and it was only after the discovery of Loeb spaces that the "lifting-up" and "pushingdown" procedures were formalized so as to rely on measure-theoretic results directly as inputs. It is worth reminding the reader that saturated spaces, and even Loeb spaces, were unknown at the time of the initial attempts at asymptotic implementation in the early seventies, and as such, we do not just traverse the old ground here. With saturated spaces, an additional consideration is added.

${ }^{37}$ From a methodological point of view, the similarity of the proof to that of Theorem 3 bears emphasis.
} 
We can now translate the existence claim in Theorem 1(ii) into an existence claim for a sequence of large but finite games with traits.

Proposition 2. Assume that the sequence of finite games $\left\{\mathcal{G}^{n}\right\}$ is tight. Then for any $\epsilon>0$, there exists $N \in \mathbb{N}$ such that for all $n \geq N$, there exists an $\epsilon$-approximate equilibrium $f^{n}$ of $\mathcal{G}^{n} .{ }^{38}$

Proof: Fix any real number $\epsilon>0$. We transfer the sequence of finite games to the nonstandard universe to obtain a sequence $\left\{\mathcal{G}^{n}\right\}_{n \in * \mathbb{N}}$ of internal games on the associated sequence of $\left\{\left(I^{n}, \mathcal{I}^{n}, \lambda^{n}\right)\right\}_{n \in * \mathbb{N}}$ of internal probability spaces. Fix any $n \in{ }^{*} \mathbb{N}_{\infty}$. Let $(I, \mathcal{I}, \lambda)$ be the corresponding Loeb space of $\left(I^{n}, \mathcal{I}^{n}, \lambda^{n}\right) .{ }^{39}$ The tightness assumption on the original sequence $\left\{\mathcal{G}^{n}\right\}_{n \in \mathbb{N}}$ implies that for each $n \in{ }^{*} \mathbb{N}_{\infty}, \mathcal{G}^{n}$ is near standard in the sense that for $\lambda$-almost all $i \in I$ and $\mathcal{G}^{n}(i)$ has a standard part ${ }^{\circ}\left(\mathcal{G}^{n}(i)\right)$ in $T \times \mathcal{V}$. For $i \in I$, let $\alpha(i)={ }^{\circ}\left(\alpha^{n}(i)\right), v_{i}={ }^{\circ}\left(v_{i}^{n}\right)$, and $\mathcal{G}(i)=\left(\alpha(i), v_{i}\right)$. Since $(I, \mathcal{I}, \lambda)$ is a saturated probability space, it follows from Theorem 1 (ii) that there exists a measurable function $f: I \longrightarrow A$ such that for $\lambda$-almost all $i \in I$,

$$
v_{i}\left(f(i), \lambda(\alpha, f)^{-1}\right) \geq v_{i}\left(a, \lambda(\alpha, f)^{-1}\right) \text { for all } a \in A
$$

By Theorem 5.2.4 in [25], we can obtain an internal lifting $f^{n}: I^{n} \longrightarrow{ }^{*} A$ of $f$ such that for $\lambda$-almost all $i \in$ $I,{ }^{\circ}\left(f^{n}(i)\right)=f(i)$. Thus, for $\lambda$-almost all $i \in I$, we have,

$$
v_{i}^{n}\left(f^{n}(i), \lambda^{n}\left(\alpha^{n}, f^{n}\right)^{-1}\right) \simeq v_{i}\left(f(i), \lambda(\alpha, f)^{-1}\right) .
$$

On the other hand, for any ${ }^{*} a \in{ }^{*} A$ and $i \in I$,

$$
v_{i}^{n}\left({ }^{*} a, \lambda^{n}\left(\alpha^{n}, f^{n}\right)^{-1}\right) \simeq v_{i}\left({ }^{\circ *} a, \lambda(\alpha, f)^{-1}\right)
$$

Comparing the above two equations with the first equation in this proof, we can assert that there exists a $I_{\epsilon}^{n} \in \mathcal{I}^{n}$ with $\lambda^{n}\left(I_{\epsilon}^{n}\right)>1-\epsilon$, such that for all $i \in I_{\epsilon}^{n}$, for all * $a \in^{*} A$,

$$
v_{i}^{n}\left(f^{n}(i), \lambda^{n}\left(\alpha^{n}, f^{n}\right)^{-1}\right) \geq v_{i}^{n}\left({ }^{*} a, \lambda^{n}\left(\alpha^{n}, f^{n}\right)^{-1}\right)-\epsilon .
$$

Since the above equation holds for all $n \in{ }^{*} N_{\infty}$, the conclusion follows the spill-over principle; for the latter, see Theorem 2.8.11 in [25].

Remark 4: Note that Proposition 2, in guaranteeing the existence of approximate equilibria for the tail of a sequence of finite games with traits, can also be seen in some sense as an approximate lower hemi-continuity result. If these approximate equilibria are approximately close to a pre-chosen equilibrium of the limit game, we have an approximate lower hemi-continuity result.

\footnotetext{
${ }^{38}$ Here we note that there is a trivial correction to be made to each of the statements of Theorems 6 and 7 in [22]. One needs to use "for all $t \in T_{\epsilon}^{n}$ with $\lambda^{n}\left(T_{\epsilon}^{n}\right)>1-\epsilon$ " instead of "for all $t \in T$ ", where $\lambda^{n}$ is the counting probability on $T^{n}$. Similarly, one needs to replace "for all $t \in T$ " in the statements of Theorems 9 and 10 by "for almost all $t \in T$ ".

${ }^{39} \mathrm{We}$ abbreviate an entire procedure here, and the interested reader can see [25] and the specific details to a game-theoretic context in [22].
} 


\section{Conclusion}

In this paper, the standard theory of large games, as surveyed in [23], and the subject of several recent extensions and applications, ${ }^{40}$ is given an alternative cast that allows a treatment of large games in which individual players have names as well as traits, and a player's dependence on society is formulated as a joint probability measure on the space of actions and traits. The key notion of a saturated player space, as first discussed in the context of the conventional formulation of the theory of large games in [17], is identified, and shown to be sufficient and necessary for a reformulated and comprehensive theory. It addresses the, by now well-known, difficulty that there is, in general, no Nash equilibrium if one works with a large game with the Lebesgue unit interval as the space of players' names and the interval $[-1,1]$ as the action space. To be sure, [22] established that this difficulty can be resolved if one works with an appropriate player space that captures the asymptotic properties of a sequence of large finite games, the Loeb counting space, but it took the suggestion of [17, Theorem 4.6] to see that with an uncountable action space, it is necessary and sufficient for a robust theory that the player space be saturated. This suggestion is now developed and placed in a broader rubric of the results presented in this paper. It is hoped that the analytically rigorous and rich formalization of traits that they shape will be more relevant in terms of applications.

\section{Appendix}

We begin this Appendix by collecting recent results on the distributions of correspondences; we present a composite result culled from the theorems in [21] and [17].

Proposition C. Let $X$ be a compact metric space and $(\Omega, \mathcal{A}, P)$ an atomless probability space. Then the following results are valid if, in addition, (i) $X$ is a countable, or (ii) $(\Omega, \mathcal{A}, P$ ) is a saturated probability space.

$P 1:$ For any correspondence $F$ from $(\Omega, \mathcal{A}, P)$ to $X, \mathcal{D}_{F}=\left\{P f^{-1}: f\right.$ is a measurable selection of $\left.F\right\}$ is convex.

$P 2$ : For any compact-valued correspondence $F$ from $(\Omega, \mathcal{A}, P)$ to $X, \mathcal{D}_{F}$ is compact.

P3: Let $F$ be a compact-valued correspondence from $(\Omega, \mathcal{A}, P)$ to $X$. Suppose that $Y$ is a metric space and $G$ is a closed-valued correspondence from $\Omega \times Y$ to $X$ such that:

(a) For all $(\omega, y) \in \Omega \times Y, G(\omega, y) \subseteq F(\omega)$.

(b) For each fixed $y \in Y, G(\cdot, y)$ (denoted by $G_{y}$ ) is a measurable correspondence from $(\Omega, \mathcal{A}, P$ ) to $X$.

(c) For each fixed $\omega \in \Omega, G(\omega, \cdot)$ is upper hemi-continuous from $Y$ to $X$.

Then the correspondence $H(y)=\mathcal{D}_{G_{y}}$ is upper hemi-continuous from $Y$ to $\mathcal{M}(X)$.

After the presentation of this basic result, we turn to the proofs.

Proof of Claim 1: (i) We first show that for any given $i, v_{i}(\cdot, \cdot)$ is a continuous function. Since $A$ has only two points, it is enough to verify the continuity with respect to $\tau$. Suppose $\left\{\tau_{n}\right\}$ converges weakly to $\tau$ in $\mathcal{M}^{\rho}(T \times A)$, where $\tau_{n} \in \mathcal{M}^{\rho}(T \times A)$ for each $n$. Let $D_{c}$ be the set of discontinuity of the function

\footnotetext{
${ }^{40}$ For the extensions of the theory, see, for example, [9] [12] and [36]; for applications, in addition to [1, 2], [8] and [10], see [5] and their references.
} 
$1_{[0, i) \times\{a\}}(t, x)$. It is clear that $\tau\left(D_{c}\right)=0$. By [6, Theorem 2.1], $\lim _{n \rightarrow \infty} v_{i}\left(a, \tau_{n}\right)=v_{i}(a, \tau)$ for any given $i$ and $a$, and thus, $v_{i}(\cdot, \cdot)$ is continuous.

Next, notice that when $i=0, v_{i}(\cdot, \cdot)$ is identically zero. We show that $v_{i}(\cdot, \cdot)$ is a continuous function of i. $v_{i}(a, \tau)=-\int_{T \times A} 1_{[0, i) \times\{a\}}(t, x) d \tau$. Assume that $j>i$. Then

$$
\begin{aligned}
v_{i}(a, \tau)-v_{j}(a, \tau) & =\int_{T \times A} 1_{[0, j) \times\{a\}}(t, x) d \tau-\int_{T \times A} 1_{[0, i) \times\{a\}}(t, x) d \tau \\
& =\int_{T \times A} 1_{[i, j) \times\{a\}}(t, x) d \tau=\tau([i, j) \times\{a\}) \leq j-i .
\end{aligned}
$$

Therefore, for any $i$ and $j$ in $I,\left|v_{i}(\cdot, \cdot)-v_{j}(\cdot, \cdot)\right| \leq|j-i|$, which shows that $v_{i}(\cdot, \cdot)$ is a continuous function of $i$. Hence, $\mathcal{G}$ is a measurable function from $I$ to $T \times \mathcal{V}_{(A, T, \rho)}$.

(ii) Assume that $f^{*}$ is a Nash equilibrium of the game $\mathcal{G}$. We first show that for all $i>0, v_{i}\left(\bar{H}, \lambda\left(\alpha, f^{*}\right)^{-1}\right)=$ $v_{i}\left(\bar{T}, \lambda\left(\alpha, f^{*}\right)^{-1}\right)$. Suppose that for some $i>0, v_{i}\left(\bar{H}, \lambda\left(\alpha, f^{*}\right)^{-1}\right)<v_{i}\left(\bar{T}, \lambda\left(\alpha, f^{*}\right)^{-1}\right)$. For this fixed $i$ let

$$
S=\left\{r \in[0, i): v_{r}\left(\bar{H}, \lambda\left(\alpha, f^{*}\right)^{-1}\right)=v_{r}\left(\bar{T}, \lambda\left(\alpha, f^{*}\right)^{-1}\right)\right\} .
$$

$S$ is nonempty since it contains 0 , so $s^{*}=\sup S$ exists. The continuity of $v_{r}(\cdot, \cdot)$ in $r$ implies that $v_{s^{*}}\left(\bar{H}, \lambda\left(\alpha, f^{*}\right)^{-1}\right)=v_{s^{*}}\left(\bar{T}, \lambda\left(\alpha, f^{*}\right)^{-1}\right)$. Note that given any $a \in A$ and any measurable function $f$ from $I$ to $A$,

$$
v_{i}\left(a, \lambda(\alpha, f)^{-1}\right)=-\int_{T \times A} 1_{[0, i) \times\{a\}}(t, x) d \lambda(\alpha, f)^{-1}=-\lambda\left([0, i) \cap f^{-1}(a)\right) .
$$

Thus, we have, $\lambda\left(\left[0, s^{*}\right) \cap f^{*-1}(\bar{H})\right)=\lambda\left(\left[0, s^{*}\right) \cap f^{*-1}(\bar{T})\right)$. Furthermore, $s^{*}<i$. For any $y \in\left(s^{*}, i\right)$, $v_{y}\left(\bar{H}, \lambda\left(\alpha, f^{*}\right)^{-1}\right)<v_{y}\left(\bar{T}, \lambda\left(\alpha, f^{*}\right)^{-1}\right)$ by the continuity of $v_{y}(\cdot, \cdot)$ in $y$. Since $f^{*}$ is a Nash equilibrium, we know $f^{*}(y)=\bar{T}$ for all $y \in\left(s^{*}, i\right)$. Therefore,

$$
\lambda\left([0, i) \cap f^{*-1}(\bar{T})\right)=\lambda\left(\left[0, s^{*}\right) \cap f^{*-1}(\bar{T})\right)+\lambda\left(\left[s^{*}, i\right) \cap f^{*-1}(\bar{T})\right)>\lambda\left(\left[0, s^{*}\right) \cap f^{*-1}(\bar{T})\right)
$$

and

$$
\lambda\left([0, i) \cap f^{*-1}(\bar{H})\right)=\lambda\left(\left[0, s^{*}\right) \cap f^{*-1}(\bar{H})\right)+\lambda\left(\left[s^{*}, i\right) \cap f^{*-1}(\bar{H})\right)=\lambda\left(\left[0, s^{*}\right) \cap f^{*-1}(\bar{H})\right) .
$$

Thus, we have

$$
v_{i}\left(\bar{H}, \lambda\left(\alpha, f^{*}\right)^{-1}\right)=-\lambda\left([0, i) \cap f^{*-1}(\bar{H})\right)>-\lambda\left([0, i) \cap f^{*-1}(\bar{T})\right)=v_{i}\left(\bar{T}, \lambda\left(\alpha, f^{*}\right)^{-1}\right),
$$

a contradiction. Similarly, for any $i>0, v_{i}\left(\bar{H}, \lambda\left(\alpha, f^{*}\right)^{-1}\right)<v_{i}\left(\bar{T}, \lambda\left(\alpha, f^{*}\right)^{-1}\right)$ cannot hold. Therefore, for every $i>0$,

$$
v_{i}\left(\bar{H}, \lambda\left(\alpha, f^{*}\right)^{-1}\right)=v_{i}\left(\bar{T}, \lambda\left(\alpha, f^{*}\right)^{-1}\right),
$$

which is equivalent to

$$
\lambda\left([0, i) \cap f^{*-1}(\bar{H})\right)=\lambda\left([0, i) \cap f^{*-1}(\bar{T})\right) .
$$


Furthermore, since $\lambda\left([0, i) \cap f^{*-1}(\bar{H})\right)+\lambda\left([0, i) \cap f^{*-1}(\bar{T})\right)=\lambda([0, i))=i$, we know that

$$
\lambda\left([0, i) \cap f^{*-1}(\bar{H})\right)=\lambda\left([0, i) \cap f^{*-1}(\bar{T})\right)=i / 2 .
$$

Let $F=\left\{i \in I: f^{*}(i)=\bar{H}\right\}$. Let $g(i)=i$ on $F, g(i)=-i$ on $I \backslash F$. Then for any $c \in(0,1], \lambda g^{-1}([0, c])=$ $\lambda g^{-1}([-c, 0])=c / 2$. So, $\lambda g^{-1}$ is the uniform distribution on $[-1,1]$. We will show that this itself is a contradiction. Clearly, $\lambda(F)>0$. Since $F$ is a subset of $[0,1], \lambda g^{-1}(F)=(1 / 2) \lambda(F)$. On the other hand, $g$ is the identity on $F$, and so, $\lambda g^{-1}(F)=\lambda(F)$. Thus, $0 \neq(1 / 2) \lambda(F)=\lambda(F)$. This contradiction establishes that the game does not have a Nash equilibrium.

The proof of Theorem 2 hinges on the proof of Lemma 2 that is provided below. This hinges on a technical construction, and to orient and motivate the reader, we first provide an argument in the form of Lemma 1 below that abstracts from traits, and is focused on a large game in its conventional form. As such, it furnishes an alternative proof ${ }^{41}$ of the necessity part of [17, Theorem 4.6], presented as Proposition B above for the convenience of the reader.

Lemma 1. Let $(I, \mathcal{I}, \lambda)$ be an atomless probability space and $A$ an uncountable, compact metric space. If every game $\mathcal{G}^{0}$ in terms of Definition 1 with player space $(I, \mathcal{I}, \lambda)$ and action space $A$ has a Nash equilibrium, then $(I, \mathcal{I}, \lambda)$ is saturated.

Proof: Consider the example in [19]. Let $\overline{\mathcal{G}}^{0}$ be a large game with the Lebesgue unit interval $(L, \mathcal{L}, l)$ as the player space and $[-1,1]$ as the action space. The payoff function $\overline{\mathcal{G}}_{j}^{0}$ of player $j \in L$ is given, for $a \in[-1,1], \rho \in \mathcal{M}([-1,1])$, by

$$
\overline{\mathcal{G}}_{j}^{0}(a, \rho)=\int_{-1}^{1} \phi_{j}(a, x) d \rho(x), \quad \text { where } \quad \phi_{j}(a, x)=-|j-| a||+(j-a) z(j, x),
$$

and the function $z:[0,1] \times[-1,1] \longrightarrow \mathbb{R}$ is such that for all $j \in[0,1]$,

$$
z(j, a)= \begin{cases}a & \text { if } 0 \leq a \leq j \\ j & \text { if } j<a \leq 1 \\ -z(j,-a) & \text { if } a<0\end{cases}
$$

As shown in [19], the game $\overline{\mathcal{G}}^{0}$ does not have a Nash equilibrium.

Suppose that $(I, \mathcal{I}, \lambda)$ is not saturated. It follows from Definition 3 that there is a set $C \in \mathcal{I}$ with $\lambda(C)=\beta, 0<\beta<1$ such that $\left(C, \mathcal{I}^{C}, \lambda^{C}\right)$ is countably-generated, where $\mathcal{I}^{C}=\{E \in \mathcal{I}, E \subseteq C\}$ and $\lambda^{C}(E)=\lambda(E) / \beta$ for all $E \in \mathcal{I}^{C}$. As in the proof of Theorem 3.7 of [17], there exists a measurable mapping $h: C \longrightarrow L$ such that $h$ induces an isomorphism from the measure algebra of $\left(C, \mathcal{I}^{C}, \lambda^{C}\right)$ to the measure algebra of $(L, \mathcal{L}, l)$ (a consequence of the well-known Maharam's theorem). Define a new large game $\hat{\mathcal{G}}^{0}$ on $(I, \mathcal{I}, \lambda)$ by letting $\hat{\mathcal{G}}_{i}^{0}(a, \rho)=\overline{\mathcal{G}}_{h(i)}^{0}(a, \rho)+(1-\beta)|h(i)-| a||$ for $i \in C$ and $\hat{\mathcal{G}}^{0}(i)(a, \rho)=1-|a|$ for $i \notin C$. Suppose that $\hat{\mathcal{G}}^{0}$ has a Nash equilibrium $g$. Then it is obvious that $g(i)=0$ for $\lambda$-almost all $i \notin C$, and there is a Borel measurable mapping $g^{0}$ from $[0,1]$ to $[-1,1]$ such that $g(i)=g^{0}(h(i))$ for $i \in C$. It is easy to

\footnotetext{
${ }^{41}$ As noted in Footnote 27 , we provide here an alternative proof that was included in an earlier unpublished version of [17]. The interested reader should gauge the complementarity in Lemma 2 and Lemma 1 by a focus on the mapping $h$, and on how it is used to transfer a counterexample on an Lebesgue interval to a general non-saturated space.
} 
check that $\lambda g^{-1}=(1-\beta) \delta_{0}+\beta l\left(g^{0}\right)^{-1}$, where $\delta_{0}$ is the probability measure on $[-1,1]$ such that $\delta_{0}(\{0\})=1$. Since $g$ is a Nash equilibrium for $\hat{\mathcal{G}}^{0}$, we know that for $\lambda$-almost all $i \in C$,

$$
\overline{\mathcal{G}}_{h(i)}^{0}\left(g(i), \lambda g^{-1}\right)+(1-\beta)|h(i)-| g(i)|| \geq \overline{\mathcal{G}}_{h(i)}^{0}\left(a, \lambda g^{-1}\right)+(1-\beta)|h(i)-| a||
$$

holds for any $a \in[-1,1]$. Since $z(j, 0)=0$ for any $j \in L$, and $\lambda g^{-1}=(1-\beta) \delta_{0}+\beta l\left(g^{0}\right)^{-1}$, we obtain that for any $a \in[-1,1]$,

$$
\overline{\mathcal{G}}_{h(i)}^{0}\left(a, \lambda g^{-1}\right)=-(1-\beta)|h(i)-| a||+\beta \overline{\mathcal{G}}_{h(i)}^{0}\left(a, l\left(g^{0}\right)^{-1}\right) .
$$

Hence, for $l$-almost all $j \in L, \overline{\mathcal{G}}_{j}^{0}\left(g^{0}(j), l\left(g^{0}\right)^{-1}\right) \geq \overline{\mathcal{G}}_{j}^{0}\left(a, l\left(g^{0}\right)^{-1}\right)$ holds for any $a \in[-1,1]$, which means that $g^{0}$ is a Nash equilibrium of $\overline{\mathcal{G}}^{0}$. This is a contradiction. Therefore, the large game $\hat{\mathcal{G}}^{0}$ has no Nash equilibrium too.

Now consider any given uncountable compact metric space $A$. As shown in [30, p.339], there exists a continuous surjective mapping $F$ from $A$ to $[-1,1]$ and a continuous injective mapping $\mathcal{F}_{0}$ from $\mathcal{U}_{[-1,1]}$ to $\mathcal{U}_{A}$ such that $\mathcal{F}_{0}(u)(x, y)=u\left(F(x), y F^{-1}\right)$ for any $u \in \mathcal{U}_{\mathcal{A}}, x \in A$ and $y \in \mathcal{M}(A)$. Let a new large game $\mathcal{G}^{0}$ with player space $(I, \mathcal{I}, \lambda)$ and action space $A$ be the composition mapping $\mathcal{G}^{0}=\mathcal{F}_{0} \circ \hat{\mathcal{G}}^{0}$. If there exists a Nash equilibrium $g^{*}$ in $\mathcal{G}^{0}$, then the composition mapping $g=F \circ g^{*}$ must be a Nash equilibrium of $\hat{\mathcal{G}}^{0}$. This is impossible. Thus, $(I, \mathcal{I}, \lambda)$ must be saturated by this contradiction.

Armed with the intuition furnished by the argument presented above we can turn to Lemma 2 and its proof.

Lemma 2. If an atomless probability space $(I, \mathcal{I}, \lambda)$ is not saturated, then there exists a large game with traits $\mathcal{G}:(I, \mathcal{I}, \lambda) \longrightarrow T \times \mathcal{V}_{(A, T, \rho)}$ that does not have a Nash equilibrium, where $A=\{\bar{H}, \bar{T}\}, T=[0,1]$ and $\rho=\lambda \mathcal{G}_{1}^{-1}$ is the Lebesgue measure on $[0,1]$.

Proof: We shall give $\left(C, \mathcal{I}^{C}, \lambda^{C}\right)$ and $\beta$ the same meaning in the proof of Lemma 1 . Let $(\hat{I}, \hat{\mathcal{I}}, \hat{\lambda})$ be a normalized Lebesgue interval where $\hat{I}=[0, \beta]$. Let $\hat{T}=[0, \beta], \hat{\alpha}(j)=j$ for all $j \in \hat{I}$ and $\hat{\rho}=\hat{\lambda} \hat{\alpha}^{-1}$. For all $j \in \hat{I}$, let $\hat{v}_{j}(a, \hat{\tau})=-\int_{\hat{T} \times A} 1_{[0, j) \times\{a\}}(t, x) d \hat{\tau}$ for all $a \in A$ and for all $\hat{\tau} \in \mathcal{M}^{\hat{\rho}}(\hat{T} \times A)$. Let $\hat{\mathcal{G}}$ be a function satisfying $\hat{\mathcal{G}}(j)=\left(\hat{\alpha}(j), \hat{v}_{j}\right)$ for all $j \in \hat{I}$. Given $(\hat{I}, \hat{\mathcal{I}}, \hat{\lambda})$ is normalized Lebesgue interval, one can check $\hat{\mathcal{G}}:(\hat{I}, \hat{\mathcal{I}}, \hat{\lambda}) \longrightarrow \mathcal{V}_{(A, \hat{T}, \hat{\rho})}$ is a large game with traits without any Nash equilibrium through Example 1. That is to say, there is no measurable function $\hat{g}: \hat{I} \longrightarrow A$, such that for $\hat{\lambda}$-almost all $j \in \hat{I}$, $\hat{v}_{j}\left(\hat{g}(j), \hat{\lambda}(\hat{\alpha}, \hat{g})^{-1}\right) \geq \hat{v}_{j}\left(a, \hat{\lambda}(\hat{\alpha}, \hat{g})^{-1}\right)$ for all $a \in A$.

Suppose that $(I, \mathcal{I}, \lambda)$ is not saturated. Similar to the proof of Lemma 1, there exists a measurable mapping $h: C \longrightarrow \hat{I}$ such that $h$ induces an isomorphism from the measure algebra of $\left(C, \mathcal{I}^{C}, \lambda^{C}\right)$ to the measure algebra of the normalized Lebesgue interval $(\hat{I}, \hat{\mathcal{I}}, \hat{\lambda})$ as a consequence of Maharam's theorem. Moreover, since it is clear that $\lambda(I \backslash C)=1-\beta$, there exists a measurable function $\alpha^{0}: I \backslash C \longrightarrow(\beta, 1]$, such that for any Borel set $B$ in $(\beta, 1], \lambda\left(\alpha^{0}\right)^{-1}(B)=l(B)$; here we use $l$ to denote the Lebesgue measure on $(\beta, 1]$. Construct a function $\alpha$ from $I$ to $T$ as follows:

$$
\alpha(i)= \begin{cases}h(i) & \text { for } i \in C \\ \alpha^{0}(i) & \text { for } i \in I \backslash C .\end{cases}
$$

It is clear that $\alpha$ is measurable. Let $\rho=\lambda \alpha^{-1}$. By construction, $\rho$ is the Lebesgue measure on $[0,1]$. Now, 
for all $a \in A$ and for all $\tau \in \mathcal{M}^{\rho}(T \times A)$, let

$$
v_{i}(a, \tau)= \begin{cases}-\int_{T \times A} 1_{[0, h(i)) \times\{a\}}(t, x) d \tau & \text { for } i \in C \\ (-1)^{1+1_{\bar{H}}(a)} & \text { for } i \in I \backslash C .\end{cases}
$$

From the proof of Claim 1, it is now easy to see that $v_{i} \in \mathcal{V}_{(A, T, \rho)}$ for any $i \in I$.

Let $\mathcal{G}(i)=\left(\alpha(i), v_{i}\right)$ for all $i \in I$. It is clear that $\mathcal{G}$ is a measurable function from $I$ to $T \times \mathcal{V}_{(A, T, \rho)}$, and thus, a large game with traits. We now show that there does not exist a Nash equilibrium in $\mathcal{G}$. Suppose that $\mathcal{G}$ has a Nash equilibrium $f^{*}: I \longrightarrow A$. It is obvious that $f^{*}(i)=\bar{H}$, for $\lambda$-almost all $i \in I \backslash C$. For $i \in C$, we first show that there exists a Borel measurable function $g$ such that $f^{*}(i)=g(h(i))$ for $\lambda$-almost all $i \in C$. Let $C_{1}=\left\{i \in C: f^{*}(i)=\bar{H}\right\}$. Since $h$ induces an isomorphism from the measure algebra of $\left(C, \mathcal{I}^{C}, \lambda^{C}\right)$ to the measure algebra of $(\hat{I}, \hat{\mathcal{I}}, \hat{\lambda})$, there exists a Borel set $B_{1} \subseteq \hat{I}$, such that, $\lambda^{C}\left(C_{1} \Delta h^{-1}\left(B_{1}\right)\right)=0$, where $\Delta$ is the symmetry difference on $\mathcal{I}^{C}$, and hence, $\lambda\left(C_{1} \Delta h^{-1}\left(B_{1}\right)\right)=0$. Thus, $g$ can be constructed as $g(i)=\bar{H}$ for $i \in B_{1}$ and $g(i)=\bar{T}$ for $i \in \hat{I} \backslash B_{1}$. It is clear that $g$ is measurable.

As $f^{*}$ is an equilibrium of $\mathcal{G}$, for $\lambda$-almost all $i \in C$, we have

$$
v_{i}\left(f^{*}(i), \lambda\left(\alpha, f^{*}\right)^{-1}\right) \geq v_{i}\left(a, \lambda\left(\alpha, f^{*}\right)^{-1}\right) \text { for all } a \in A,
$$

which is to say, for $\lambda$-almost all $i \in C$,

$$
-\int_{T \times A} 1_{[0, h(i)) \times\left\{f^{*}(i)\right\}}(t, x) d \lambda\left(\alpha, f^{*}\right)^{-1} \geq-\int_{T \times A} 1_{[0, h(i)) \times\{a\}}(t, x) d \lambda\left(\alpha, f^{*}\right)^{-1} \text { for all } a \in A .
$$

Because $1_{[0, h(i)) \times\left\{f^{*}(i)\right\}}$ is zero on $(\beta, 1] \times A$, the above equation can be written as

$$
-\int_{[0, \beta] \times A} 1_{[0, h(i)) \times\left\{f^{*}(i)\right\}}(t, x) d \lambda\left(\alpha, f^{*}\right)^{-1} \geq-\int_{[0, \beta] \times A} 1_{[0, h(i)) \times\{a\}}(t, x) d \lambda\left(\alpha, f^{*}\right)^{-1} \text { for all } a \in A .
$$

Dividing both sides of the above equation by $\beta$ and thus, normalizing, we have for $\lambda$-almost all $i \in C$,

$$
\hat{v}_{h(i)}\left(g(h(i)), \lambda^{C}(h, g(h(i)))^{-1}\right) \geq \hat{v}_{h(i)}\left(a, \lambda^{C}(h, g(h(i)))^{-1}\right) \text { for all } a \in A .
$$

Hence, for $\hat{\lambda}$-almost all $j \in \hat{I}$, the measurable function $g$ satisfies

$$
\hat{v}_{j}\left(g(j), \hat{\lambda}(\hat{\alpha}, g)^{-1}\right) \geq \hat{v}_{j}\left(a, \hat{\lambda}(\hat{\alpha}, g)^{-1}\right) \text { for all } a \in A .
$$

This is a contradiction to the last sentence of the first paragraph. Therefore, the constructed game $\mathcal{G}$ does not have a Nash equilibrium.

Finally, we turn to the proof of Lemma 3 which is an essential ingredient in the proof of Theorem 2.

Lemma 3. If an atomless probability space $(I, \mathcal{I}, \lambda)$ is not saturated and $T^{\prime}$ is uncountable, complete, separable metric space with an atomless probability measure $\rho^{\prime}$, then there exists a large game with traits $\mathcal{G}^{\prime}: I \longrightarrow T^{\prime} \times \mathcal{V}_{\left(A, T^{\prime}, \rho^{\prime}\right)}$ where $A=\{\bar{H}, \bar{T}\}$, that has no Nash equilibrium.

Proof: Throughout this proof, we shall follow the notation in the proof of Lemma 2. Consider any given 
uncountable complete separable metric space $T^{\prime}$ endowed with an atomless probability measure $\rho^{\prime}$ on the Borel $\sigma$-algebra $\mathcal{B}\left(T^{\prime}\right)$ induced by the topology on $T^{\prime}$. By Theorem 1 in [33], there exists a Borel measurable bijection $F: T^{\prime} \longrightarrow T$ such that $F$ is measure preserving between $\left(T^{\prime}, \mathcal{B}\left(T^{\prime}\right), \rho^{\prime}\right)$ and $(T, \mathcal{B}(T), \rho)$, and continuous $\rho^{\prime}$-almost everywhere, and $F^{-1}$ is continuous $\rho$-almost everywhere. Now consider a mapping $\mathcal{G}^{\prime}=\left(\alpha^{\prime}, v^{\prime}\right)$ which satisfies that $\alpha^{\prime}=F^{-1}(\alpha)$ and for each $i \in I, v_{i}^{\prime}\left(a, \tau^{\prime}\right)=v_{i}\left(a, \tau^{\prime}\left(F, i d_{A}\right)^{-1}\right)$ for all $a \in A$ and $\tau^{\prime} \in \mathcal{M}^{\rho^{\prime}}\left(T^{\prime} \times A\right)$, where $i d_{A}$ stands for the identity map on $A$. In order to show $\mathcal{G}^{\prime}$ is a well-defined large game with traits, we need to show that for any given $a \in A, v_{i}^{\prime}(a, \cdot)$ is continuous for any $i$. Suppose that a sequence $\left\{{\tau^{\prime \prime}}^{n}\right\}$ in $\mathcal{M}^{\rho^{\prime}}\left(T^{\prime} \times A\right)$ converges weakly to ${\tau^{\prime}}^{0} \in \mathcal{M}^{\rho^{\prime}}\left(T^{\prime} \times A\right)$. Since $F$ is continuous $\rho^{\prime}$ almost everywhere, $\left(F, i d_{A}\right)$ is continuous ${\tau^{\prime}}^{0}$-almost everywhere. Hence, $\left\{{\tau^{\prime \prime}}^{n}\left(F, i d_{A}\right)^{-1}\right\}$ converges weakly to ${\tau^{\prime}}^{0}\left(F, i d_{A}\right)^{-1}$ as well. Therefore, for any $a \in A$, the continuity of $v_{i}(a, \cdot)$ implies $v_{i}^{\prime}(a, \cdot)$ is continuous for any $i$. Thus, $\mathcal{G}^{\prime}$ is a large game with traits.

We now show that the game $\mathcal{G}^{\prime}$ does not have an equilibrium. Suppose it does have an equilibrium, say, $f^{\prime}$. Then, for $\lambda$-almost all $i$,

$$
\begin{gathered}
v_{i}^{\prime}\left(f^{\prime}(i), \lambda\left(\alpha^{\prime}, f^{\prime}\right)^{-1}\right) \geq v_{i}^{\prime}\left(a, \lambda\left(\alpha^{\prime}, f^{\prime}\right)^{-1}\right) \text { for all } a \in A, \\
v_{i}^{\prime}\left(f^{\prime}(i), \lambda\left(F^{-1}(\alpha), f^{\prime}\right)^{-1}\right) \geq v_{i}^{\prime}\left(a, \lambda\left(F^{-1}(\alpha), f^{\prime}\right)^{-1}\right) \text { for all } a \in A .
\end{gathered}
$$

Hence, we have for $\lambda$-almost all $i$,

$$
v_{i}\left(f^{\prime}(i), \lambda\left(\alpha, f^{\prime}\right)^{-1}\right) \geq v_{i}\left(a, \lambda\left(\alpha, f^{\prime}\right)^{-1}\right) \text { for all } a \in A .
$$

That is to say, $f^{\prime}$ is a Nash equilibrium of the game $\mathcal{G}$ in Lemma 2. This is a contradiction. Therefore, the constructed game $\mathcal{G}^{\prime}$ does not have any equilibrium.

\section{References}

[1] G.A. Akerlof and R.E. Kranton, Economics and identity, Quarterly Journal of Economics 115 (2000), $715-753$.

[2] G.A. Akerlof and R.E. Kranton, Identity and schooling: some lessons for the economics of education, Journal of Economic Literature 40 (2002), 1167-1201.

[3] C.D. Aliprantis and K.C. Border, Infinite Dimensional Analysis: a Hitchhiker's Guide, 3rd edition, Springer-Verlag, Berlin, 2007.

[4] R.M. Anderson, The core in perfectly competitive economies, in: R.J. Aumann and S. Hart (Eds.), Handbook of Game Theory, Vol.2, North-Holland, New York, 1992, pp. 413-457.

[5] G.-M. Angeletos, C. Hellwig and A. Pavan, Dynamic global games of regime change: learning, multiplicity and the timing of attacks, Econometrica $\mathbf{7 5}$ (2007), 711-756.

[6] P. Billingsley, Convergence of Probability Measures, 2nd edition, Wiley-Interscience, New York, 1999.

[7] L.E. Blume, W.A. Brock, S.N. Durlauf and Y.M. Ioannides, Identification of social interactions, In: J. Benhabib, A. Bisin, and M. Jackson (Eds.), Handbook of Social Economics, Vol.1, Elsevier Science \& Technology Books, Amsterdam, 2010, pp. 855-966.

[8] W.A. Brock and S.N. Durlauf, Discrete choice with social interactions, Review of Economic Studies 68 (2001), 235-260. 
[9] G. Carmona, Large games with countable characteristics, Journal of Mathematical Economics 44 (2008), $344-347$.

[10] S.N. Durlauf and Y.M. Ioannides, Social interactions, Annual Review of Economics 2 (2010), $451-478$.

[11] S. Fajardo and H.J. Keisler, Model Theory of Stochastic Processes: Lecture Notes in Logic, Vol.14. Association for Symbolic Logic, Urbana, 2002.

[12] H. Fu, Equilibria of large games and Bayesian games with private and public information, Unpublished Ph. D. Dissertation, Department of Statistics, National University of Singapore, 2008.

[13] D.N. Hoover and H.J. Keisler, Adapted probability distributions, Transactions of the American Mathematical Society 286 (1984), 159-201.

[14] T.H. Huxley, Evolution and Ethics, Macmillan, London, 1893.

[15] M.O. Jackson, Social and Economic Networks, Princeton University Press, Princeton, 2010.

[16] S. Kakutani, Construction of a non-separable extension of the Lebesgue measure space, Proceedings of the imperial Academy 20 (1944), 115-119.

[17] H.J. Keisler and Y.N. Sun, Why saturated probability spaces are necessary, Advances in Mathematics 221 (2009), 1584-1607.

[18] M.A. Khan, On Cournot-Nash equilibrium distributions for games with a nonmetrizable action space and upper semicontinuous payoffs, Transactions of the American Mathematical Society 315 (1989), 127146 .

[19] M.A. Khan, K.P. Rath, Y.N. Sun, Pure-strategy Nash equilibrium points in large non-anonymous games, in: B. Dutta, T. Parthasarathy, J. Potters, T. E. S. Raghavan, D. Ray, and A. Sen (Eds.), Game Theoretical Applications to Economics and Operations Research, Kluwer Academic Publishers, Amsterdam, 1997, pp. 113-127.

[20] M.A. Khan, K.P. Rath and Y.N. Sun, The Dvoretzky-Wald-Wolfowitz theorem and purification in atomless finite-action games, International Journal of Game Theory 34 (2006), 91-104.

[21] M.A. Khan and Y.N. Sun, Pure strategies in games with private information, Journal of Mathematical Economics 24 (1995), 633-653.

[22] M.A. Khan and Y.N. Sun, Non-cooperative games on hyperfinite Loeb spaces, Journal of Mathematical Economics 31 (1999), 455-492.

[23] M.A. Khan and Y.N. Sun, Non-cooperative games with many players, in: R.J. Aumann and S. Hart (Eds), Handbook of Game Theory with Economic Applications vol. 3, Elsevier Science, Amsterdam, 2002, pp. 1761-1808.

[24] M.A. Khan and Y. Zhang, Set-valued functions, Lebesgue extensions and saturated probability spaces, Advances in Mathematics, 229 (2012), 1080-1103.

[25] P.A. Loeb and M. Wolff, eds. Nonstandard Analysis for the Working Mathematician, Kluwer Academic Publishers, Dordrecht, 2000.

[26] A. Mas-Colell, On a theorem of Schmeidler, Journal of Mathematical Economics 13 (1984), 201-206.

[27] K. Podczeck, On the convexity and compactness of the integral of a Banach space valued correspondence, Journal of Mathematical Economics 44 (2008), 836-852.

[28] S. Rashid, Equilibrium points of non-atomic games: asymptotic results, Economics Letters 12 (1983), $7-10$.

[29] K.P. Rath, Existence and upper hemicontinuity of equilibrium distributions of anonymous games with discontinuous payoffs, Journal of Mathematical Economics 26 (1996), 305-324. 
[30] K.P. Rath, Y.N. Sun and S. Yamashige, The nonexistence of symmetric equilibria in anonymous games with compact action spaces, Journal of Mathematical Economics 24 (1995), 331-346.

[31] D.J. Roberts and A. Postlewaite, The incentives for price-taking behavior in large exchange economies, Econometrica 44 (1976), 115-127.

[32] D. Schmeidler, Equilibrium points of non-atomic games, Journal of Statistical Physics 7 (1973), 295300 .

[33] Y.N. Sun, Isomorphisms for convergence structures, Advances in Mathematics 116 (1995), 322-355.

[34] Y.N. Sun, The exact law of large numbers via Fubini extension and characterization of insurable risks, Journal of Economic Theory 126 (2006), 31-69.

[35] Y.N. Sun and Y. Zhang, Individual risk and Lebesgue extension without aggregate uncertainty, Journal of Economic Theory 144 (2009), 432-443.

[36] H. Yu and Z. Zhang, Pure strategy equilibria in games with countable actions, Journal of Mathematical Economics 43 (2007), 192-200. 